\title{
PERIOD POLYNOMIALS AND EXPLICIT FORMULAS FOR HECKE OPERATORS ON $\Gamma_{0}(2)$
}

\author{
SHINJI FUKUHARA AND YIFAN YANG
}

\begin{abstract}
Let $S_{w+2}\left(\Gamma_{0}(N)\right)$ be the vector space of cusp forms of weight $w+2$ on the congruence subgroup $\Gamma_{0}(N)$. We first determine explicit formulas for period polynomials of elements in $S_{w+2}\left(\Gamma_{0}(N)\right)$ by means of Bernoulli polynomials. When $N=2$, from these explicit formulas we obtain new bases for $S_{w+2}\left(\Gamma_{0}(2)\right)$, and extend the Eichler-Shimura-Manin isomorphism theorem to $\Gamma_{0}(2)$. This implies that there are natural correspondences between the spaces of cusp forms on $\Gamma_{0}(2)$ and the spaces of period polynomials. Based on these results, we will find explicit form of Hecke operators on $S_{w+2}\left(\Gamma_{0}(2)\right)$. As an application of our main theorems, we will also give an affirmative answer to a speculation of Imamoglu and Kohnen on a basis of $S_{w+2}\left(\Gamma_{0}(2)\right)$.
\end{abstract}

\section{Introduction}

Let $\Gamma$ be a congruence subgroup of $S L_{2}(\mathbb{Z})$. One of the most important problems in the theory of modular forms is to obtain explicit formulas for Hecke operators on cusp forms for $\Gamma$. When $\Gamma$ is the full modular group $S L_{2}(\mathbb{Z})$, this was done in [8], where we gave explicit formulas in terms of Bernoulli numbers $B_{k}$ and divisor functions $\sigma_{k}$. Here we briefly recall the approach in [8].

For a cusp form $f$ of weight $w+2$ on $S L_{2}(\mathbb{Z})$ with $w \geq 2$ even we consider the $n$-th period

$$
r_{n}(f)=\int_{0}^{i \infty} f(z) z^{n} d z, \quad 0 \leq n \leq w .
$$

Since $r_{n}: S_{w+2}\left(S L_{2}(\mathbb{Z})\right) \rightarrow \mathbb{C}$ is a linear functional, there exists a unique cusp form $R_{n}$ of weight $w+2$ such that $r_{n}(f)=\left(f, R_{n}\right)$, where $(f, g)$ is the Petersson inner product. In [8], we first showed that a certain subsets of $\left\{R_{n}\right\}$ forms a basis for the space $S_{w+2}\left(S L_{2}(\mathbb{Z})\right)$ of cusp forms of weight $w+2$. We then studied the action of Hecke operators on this basis and obtained an explicit matrix representation of Hecke operators. The Dedekind symbols (9]) played a central role in our argument. (Note that periods and the Hecke operators on periods have been studied by a number of mathematicians. To name a few, see [1, 5, 8, 12, 14, 15, 17, 18, 19, 20.)

The main goal in this article is to extend our formulas from $S L_{2}(\mathbb{Z})$ to $\Gamma_{0}(N)$. Our starting point is the same as the case for $S L_{2}(\mathbb{Z})$. Namely, for a cusp form $f$ of weight $w+2$ on $\Gamma_{0}(N)$, we consider the $n$-th period $r_{n}(f)=\int_{0}^{i \infty} f(z) z^{n} d z$ for $0 \leq n \leq w$, and let $R_{\Gamma_{0}(N), w, n}$ be the unique cusp form determined by the property

$$
r_{n}(f)=2^{-1}(2 i)^{w+1}\left(f, R_{\Gamma_{0}(N), w, n}\right)
$$

2000 Mathematics Subject Classification. Primary 11F25; Secondary 11F11, 11F67.

Key words and phrases. Hecke operators, modular forms (one variable), period polynomials. 
for all cusp forms $f \in S_{w+2}\left(\Gamma_{0}(N)\right)$. (The choice of the constant $2^{-1}(2 i)^{w+1}$ is to make our formulas look nicer.) However, for lack of Dedekind symbols on $\Gamma_{0}(N)$, the method for $S L_{2}(\mathbb{Z})$ in $[8$, would not work here, and we ought to develop a new method to handle the case $\Gamma_{0}(N)$.

First of all, it is known in literature that these cusp forms $R_{\Gamma_{0}(N), w, n}$ can be expressed in terms of certain Poincaré series. We then observe that with those Poincaré series representation, the action of Hecke operators on $R_{\Gamma_{0}(N), w, n}$ can be described very concretely. This observation turns out to be crucial in our method. This is because if a basis in terms of $R_{\Gamma_{0}(N), w, n}$ exists, then we can compute the periods of $R_{\Gamma_{0}(N), w, n}$ and those of $T_{m} R_{\Gamma_{0}(N), w, n}$ to get matrix representations of Hecke operators $T_{m}$. This basically summarizes our approach. However, here we should remark that for a general integer $N$, the set $\left\{R_{\Gamma_{0}(N), w, n}\right\}$ cannot possibly span the whole space. This is because the dimension of $S_{w+2}\left(\Gamma_{0}(N)\right)$ is roughly $w\left[S L_{2}(\mathbb{Z}): \Gamma_{0}(N)\right] / 12$, while we have only $w+1$ different $R_{\Gamma_{0}(N), w, n}$. For general integers, one might need to consider more general periods. (See [1, 5, 18.) Furthermore, for $m$ and $n$ with the same parity, not much can be said about $r_{m}\left(R_{\Gamma_{0}(N), w, n}\right)$. Thus, for odd $n$, we consider only even periods, and for even $n$, we consider only odd periods. Consequently, we will only consider the cases where $\left[S L_{2}(\mathbb{Z}): \Gamma_{0}(N)\right] \leq 6$, that is, $N=2, \ldots, 5$.

For the case $N=2$ we are able to determine a basis for every even weight $w+2$. To achieve this, we show that the images of the period maps are linearly independent for certain subsets of $\left\{R_{\Gamma_{0}(2), w, n}\right\}$. Then we obtain explicit formulas for Hecke operators by computing the periods of $T_{m} R_{\Gamma_{0}(2), w, n}$. As a consequence of knowing an explicit basis for $S_{w+2}\left(\Gamma_{0}(2)\right)$, we can establish the $\Gamma_{0}(2)$-version of the Eichler-Shimura-Manin theorem ([7, [12, [15, [17), which strengthens a result of Imamoḡlu and Kohnen ([10, Proposition 3]. Furthermore, we give an affirmative answer to a speculation raised by Imamoglu and Kohnen [10, which is described as follows.

For even integers $k \geq 4$, let

$$
E_{k}^{i \infty}(z)=\frac{1}{2} \sum_{\operatorname{gcd}(c, d)=1,2 \mid c} \frac{1}{(c z+d)^{k}}, \quad E_{k}^{0}(z)=\frac{1}{2} \sum_{\operatorname{gcd}(c, d)=1,2 \nmid c} \frac{1}{(c z+d)^{k}}
$$

be normalized Eisenstein series of weight $k$ for the cusps $i \infty$ and 0 , respectively. It is obvious that $E_{2 j}^{i \infty} E_{k-2 j}^{0}, j=2,3, \ldots, k / 2-2$, are all cusp forms of weight $k$ on $\Gamma_{0}(2)$. Inspired by a conjecture of Chan and Chua [2, Imamoḡlu and Kohnen [10, Theorem 1] proved that these forms generate the whole space $S_{k}\left(\Gamma_{0}(2)\right)$ of cusp forms of weight $k$ on $\Gamma_{0}(2)$. The number of such forms exceeds the dimension of $S_{k}\left(\Gamma_{0}(2)\right)$ when $k>8$, so it is natural to determine which functions form a basis for $S_{k}\left(\Gamma_{0}(2)\right)$. The experimental calculation in 10 suggests that the first few functions $E_{2 j}^{i \infty} E_{k-2 j}^{0}, j=2,3, \ldots$, will constitute a basis. In this paper, we will prove that this is indeed true in general as a corollary of our main theorems.

For the cases $N=3,4,5$, we are unable to obtain analogous results, although our numerical computation suggests that the first $d_{w}$ cusp forms $R_{\Gamma_{0}(N), w, 2 i}, i=$ $1, \ldots, d_{w}=\operatorname{dim} S_{w+2}\left(\Gamma_{0}(N)\right)$, are always a basis for $S_{w+2}\left(\Gamma_{0}(N)\right)$. In any case, as long as a basis in terms of $R_{\Gamma_{0}(N), w, n}$ is found, we can use our formulas for period polynomials to obtain matrices for Hecke operators. 


\section{Definitions And Statements of Results}

Throughout the paper, we assume that $N$ is an integer with $N>1$ and $w$ is an even positive integer. For a cusp form $f \in S_{w+2}\left(\Gamma_{0}(N)\right)$ of weight $w+2$ on $\Gamma_{0}(N)$, we let

$$
r_{n}(f):=\int_{0}^{i \infty} f(z) z^{n} d z
$$

be the $n$-th period of $f$, and let the period polynomial $r(f)$ be defined by

$$
r(f)(X):=\int_{0}^{i \infty} f(z)(X-z)^{w} d z .
$$

Furthermore, even and odd period polynomials $r^{+}(f)$ and $r^{-}(f)$ are defined by

If we set

$$
r^{ \pm}(f)(X):=\frac{1}{2}\{r(f)(X) \pm r(f)(-X)\}
$$

$V_{w}:=$ the vector space of polynomials of degree $\leq w$ in one variable $X$, then we have homomorphisms of vector spaces:

$$
\begin{gathered}
r: S_{w+2}\left(\Gamma_{0}(N)\right) \rightarrow V_{w}, \\
r^{ \pm}: S_{w+2}\left(\Gamma_{0}(N)\right) \rightarrow V_{w} .
\end{gathered}
$$

Hereafter, $B_{m}(x)$ (resp. $B_{m}$ ) denotes the $m$-th Bernoulli polynomial (resp. number). By $B_{m}^{0}(x)$, we denote the $m$-th Bernoulli polynomial without its $B_{1}$-term ([12, p. 208]):

$$
B_{m}^{0}(x):=\sum_{\substack{0 \leq i \leq m \\
i \neq 1}}\left(\begin{array}{c}
m \\
i
\end{array}\right) B_{i} x^{m-i}=\sum_{\substack{0 \leq i \leq m \\
i \text { even }}}\left(\begin{array}{c}
m \\
i
\end{array}\right) B_{i} x^{m-i}
$$

Moreover, $\operatorname{sgn}(x)$ denotes the sign of $x \in \mathbb{R}$. For an integer $n$ with $0 \leq n \leq w$, let $\tilde{n}$ stand for $w-n$.

First we need the following definitions to state our results:

Definition 1.1. (1) For an integer $n$ such that $0 \leq n \leq w$, we let $R_{\Gamma_{0}(N), w, n}(z)$ be the unique cusp form of weight $w+2$ on $\Gamma_{0}(N)$ characterized by

$$
2^{-1}(2 i)^{w+1}\left(f, R_{\Gamma_{0}(N), w, n}\right)=\int_{0}^{i \infty} f(z) z^{n} d z=r_{n}(f)
$$

for all cusp forms $f$ of the same weight on $\Gamma_{0}(N)$, where

$$
(f, g)=\iint_{\Gamma_{0}(N) \backslash \mathbb{H}} f(z) \overline{g(z)} y^{w} d x d y, \quad z=x+i y,
$$

denotes the Petersson inner product of $f$ and $g$;

(2) For an integer $n$ with $0<n<w$, we define a polynomial $S_{N, w, n}$ in $X$ by

$$
S_{N, w, n}(X):=\frac{N^{\tilde{n}} X^{w}}{\tilde{n}+1} B_{\tilde{n}+1}^{0}\left(\frac{1}{N X}\right)-\frac{1}{n+1} B_{n+1}^{0}(X) .
$$

The reader should be advised that our definition of the Petersson inner product differs from the standard definition by a factor $\left[S L_{2}(\mathbb{Z}): \Gamma_{0}(N)\right]$. This is for the sake of making the presentations simpler.

In our first theorem we evaluate the period polynomials of $R_{\Gamma_{0}(N), w, n}$. This result is crucial in our subsequent discussion. 
Theorem 1.1. Let $N$ be an integer greater than 1 . For an even integer $n$ with $0<n<w$, we have

$$
r^{-}\left(R_{\Gamma_{0}(N), w, n}\right)(X)=S_{N, w, n}(X) .
$$

Also, for an odd integer $n$ with $0<n<w$, we have

$$
\begin{aligned}
& r^{+}\left(R_{\Gamma_{0}(N), w, n}\right)(X)=S_{N, w, n}(X) \\
& \quad-\frac{(w+2) B_{n+1} B_{\tilde{n}+1}}{(n+1)(\tilde{n}+1) B_{w+2}}\left(\frac{X^{w}}{N} \prod_{p \mid N} \frac{1-p^{-(n+1)}}{1-p^{-(w+2)}}-\frac{1}{N^{n+1}} \prod_{p \mid N} \frac{1-p^{-(\tilde{n}+1)}}{1-p^{-(w+2)}}\right),
\end{aligned}
$$

where $p$ runs over all prime divisors of $N$.

We remark that the formulas for period polynomials for the case $N>1$ is actually simpler than those for the case $N=1$ given in [12. This is because matrices in $\Gamma_{0}(N)$ with $N>1$ cannot have zeroes at the $(1,1)$-entry and the $(2,2)$-entry. We note also that the formula for the period polynomial $r^{+}\left(R_{\Gamma_{0}(N), w, n}\right)(X), n$ odd, has already been discussed in [1. However, the discussion in [1] is in a very broad setting, and consequently, the formulas of [1] are too complicated to be applied readily to our situation.

We now devote our attention to the study of the action of Hecke operators on $R_{\Gamma_{0}(N), w, n}$. For this purpose, we will need the following definitions:

Definition 1.2. (1) For a positive integer $m$, let

$H_{N, m}:=\left\{\left[\begin{array}{ll}a & b \\ c & d\end{array}\right] \mid a, b, c, d \in \mathbb{Z} ; a d-b c=m ; c \equiv 0 \quad(\bmod N) ; \operatorname{gcd}(a, N)=1\right\} ;$

(2) For positive integers $m$ and $n$ such that $0<n<w$, let

$$
\begin{aligned}
& R_{\Gamma_{0}(N), w, n}^{m}(z) \\
& \quad:=m^{w+1} c_{w, n}^{-1} \sum_{\left[\begin{array}{ll}
a & b \\
c & d
\end{array}\right] \in H_{N, m}} \frac{1}{(a z+b)^{\tilde{n}+1}(c z+d)^{n+1}}, c_{w, n}=(-1)^{n} 2 \pi i\left(\begin{array}{l}
w \\
n
\end{array}\right) ;
\end{aligned}
$$

(3) For positive integers $m$ and $n$ such that $0<n<w$, we define a polynomial $S_{N, w, n}^{m}$ in $X$ by

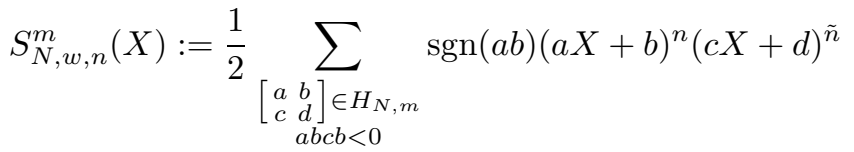

$$
\begin{aligned}
& +\sum_{\substack{a d=m, a>0 \\
\operatorname{gcd}(a, N)=1}}\left\{\frac{a^{n} N^{\tilde{n}} X^{w}}{\tilde{n}+1} B_{\tilde{n}+1}^{0}\left(\frac{d}{N X}\right)-\frac{d^{\tilde{n}}}{n+1} B_{n+1}^{0}(a X)\right\} .
\end{aligned}
$$

Then the actions of Hecke operators on $R_{\Gamma_{0}(N), w, n}$ are explicitly as follows:

Lemma 1.2. Let $m$ be a positive integer, the action of the Hecke operators $T_{m}$ on $R_{\Gamma_{0}(N), w, n}$ is

$$
T_{m}\left(R_{\Gamma_{0}(N), w, n}\right)=R_{\Gamma_{0}(N), w, n}^{m} .
$$

We remark that Lemma 1.2 for the case $N=1$ is casually mentioned in [12] without a proof. For the convenience of the reader we will provide a proof in Section 3 
From this series expression of $T_{m}\left(R_{\Gamma_{0}(N), w, n}\right)$, we can compute the period polynomials of $T_{m}\left(R_{\Gamma_{0}(N), w, n}\right)$ for even $n$.

Theorem 1.3. Let $n$ be an even integer with $0<n<w$. If $m$ is a positive integer not divisible by $N$, then

$$
r^{-}\left(R_{\Gamma_{0}(N), w, n}^{m}\right)(X)=S_{N, w, n}^{m}(X) .
$$

Furthermore, when $m$ is a multiple of $N$, we have

$$
r^{-}\left(R_{\Gamma_{0}(N), w, n}^{m}\right)(X)=S_{N, w, n}^{m}(X)-\frac{(N X)^{w}}{n+1} \sum_{d \mid N} \frac{\mu(N / d)}{d^{n}} \sum_{c \mid(m / N)} c^{\tilde{n}} B_{n+1}^{0}\left(\frac{m d}{c N^{2} X}\right),
$$

where $\mu(d)$ is the Möbius function.

\section{Examples.}

(1) Let $N=4, w=4$ and consider the Hecke operator $T_{2}$ on $S_{6}\left(\Gamma_{0}(4)\right)$. Since $T_{2}$ sends a cusp form on $\Gamma_{0}(4)$ to a cusp form on $\Gamma_{0}(2)$ and $\operatorname{dim} S_{6}\left(\Gamma_{0}(2)\right)=$ 0 , we should have $S_{4,4,2}^{2}(X)=0$. Indeed, we have

$$
\begin{aligned}
S_{4,4,2}^{2}(X) & =\frac{4^{2} X^{4}}{3} B_{3}^{0}\left(\frac{2}{4 X}\right)-\frac{2^{2}}{3} B_{3}^{0}(X) \\
& =\frac{16 X^{4}}{3}\left(\frac{1}{8 X^{3}}+\frac{3}{6} \frac{1}{2 X}\right)-\frac{4}{3}\left(X^{3}+\frac{3}{6} X\right)=0 .
\end{aligned}
$$

(2) Let $N=4, w=6$, and consider the Hecke operator $T_{8}=T_{2}^{3}$. Then $T_{8} R_{\Gamma_{0}(4), 6,2}$ is a scalar multiple of $R_{\Gamma_{0}(2), 6,2}$ since $\operatorname{dim} S_{8}\left(\Gamma_{0}(2)\right)=1$. Thus, the period polynomial $r^{-}\left(R_{\Gamma_{0}(4), 6,2}^{8}\right)(X)$ should be a scalar multiple of $r^{-}\left(R_{\Gamma_{0}(2), 6,2}\right)(X)$. We have

$$
S_{2,6,2}(X)=\frac{2^{4} X^{6}}{5} B_{5}^{0}\left(\frac{1}{2 X}\right)-\frac{1}{3} B_{3}^{0}(X)=-\frac{1}{15}\left(4 X^{5}-5 X^{3}+X\right) .
$$

For $r^{-}\left(R_{\Gamma_{0}(4), 6,2}^{8}\right)$, we find that there are 4 tuples $(a, b, c, d)= \pm(1,1,-4,4)$ and $\pm(1,-1,4,4)$ contributing to the first sum in $S_{4,6,2}^{8}(X)$, giving

$$
-1024\left(X^{5}-2 X^{3}+X\right)
$$

while the remaining part of $S_{4,6,2}^{8}(X)$ is

$$
\frac{4^{4} X^{6}}{5} B_{5}^{0}(2 / X)-\frac{8^{4}}{3} B_{3}^{0}(X)=-\frac{256}{15}\left(X^{5}+40 X^{3}-56 X\right) .
$$

The other term in $r^{-}\left(R_{\Gamma_{0}(4), 6,2}^{8}\right)$ is

$$
-\frac{(4 X)^{6}}{3} \sum_{d \mid 4} \mu(4 / d) d^{-2} \sum_{c \mid 2} c^{4} B_{3}^{0}(d /(2 c X))=256\left(3 X^{5}-4 X^{3}\right),
$$

and we find

$$
r^{-}\left(R_{\Gamma_{0}(4), 6,2}^{8}\right)(X)=-\frac{1024}{15}\left(4 X^{5}-5 X^{3}+X\right),
$$

which is indeed a multiple of $r^{-}\left(R_{\Gamma_{0}(2), 6,2}\right)(X)$. 
Remark. Observant readers will notice that when the space $S_{w+2}\left(\Gamma_{0}(N)\right)$ has dimension 1 , we have

$$
R_{\Gamma_{0}(N), w, n}^{m}=a_{m} R_{\Gamma_{0}(N), w, n},
$$

where $a_{m}$ is the eigenvalue of the $m$-th Hecke operator. Thus, by considering the coefficients of the period polynomials of both sides, we can obtain explicit expressions for $a_{m}$. For example, take $N=2, w=6$. If we choose $n=2$, then Theorem 1.1 yields

$$
r^{-}\left(R_{\Gamma_{0}(2), 6,2}\right)(X)=N^{3} B_{4} X^{5}+\cdots,
$$

while Lemma 1.2 and Theorem 1.3 give, for $2 \nmid m$,

$$
\begin{aligned}
r^{-}\left(T_{m} R_{\Gamma_{0}(2), 6,2}\right)= & X^{5} N^{3} B_{4} \sum_{a d=m} a^{2} d \\
& +2 X^{5} \sum_{\substack{a, b, c, d>0 \\
a d+2 b c=m}}\left(2 a b(2 c)^{4}-4 a^{2}(2 c)^{3} d\right)+\cdots .
\end{aligned}
$$

Thus, for $2 \nmid m$, we see that the $m$-th Hecke eigenvalue, is

$$
m \sum_{a \mid m} a+240 \sum_{\begin{array}{c}
a, b, c, d>0 \\
a d+2 b c=m
\end{array}} a c^{3}(a d-b c)=m \sigma_{1}(m)+240 \sum_{u+2 v=m}(u-v) \sigma_{1}(u) \sigma_{3}(v) .
$$

Although this formula looks like an interesting application of period polynomials, it, in fact, can be more easily obtained by applying the Rankin-Cohen bracket (see [3, 16]) to the pair of modular forms

$$
1-24 \sum_{n=1}^{\infty} n\left(\frac{2 q^{2 n}}{1-q^{2 n}}-\frac{q^{n}}{1-q^{n}}\right), \quad 1+240 \sum_{n=1}^{\infty} \frac{n^{3} q^{2 n}}{1-q^{2 n}}, \quad\left(q=e^{2 \pi i z}\right)
$$

on $\Gamma_{0}(2)$.

In the remainder of the section, we restrict our attention to the case $N=2$. In this case we are able to determine bases for $S_{w+2}\left(\Gamma_{0}(2)\right)$. For the sake of convenience, we set

$$
\left.d_{w}:=\left\lfloor\frac{w-2}{4}\right\rfloor \text { (i.e., } d_{w} \text { is the greatest integer not exceeding }(w-2) / 4\right) .
$$

It is well-known (see e.g. [6]) that

$$
\operatorname{dim} S_{w+2}\left(\Gamma_{0}(2)\right)=d_{w} .
$$

By applying Theorem 1.1 with $N=2$ and showing the linear independence among the period polynomials of $R_{\Gamma_{0}(2), w, n}$ for certain ranges of integers $n$, we can determine a basis for $S_{w+2}\left(\Gamma_{0}(2)\right)$.

Theorem 1.4. Each of the sets

$$
\left\{R_{\Gamma_{0}(2), w, 2 i} \mid i=1,2, \ldots, d_{w}\right\}, \quad\left\{R_{\Gamma_{0}(2), w, w-2 i} \mid i=1,2, \ldots, d_{w}\right\}
$$

and

$$
\left\{R_{\Gamma_{0}(2), w, 2 i-1} \mid i=1,2, \ldots, d_{w}\right\}, \quad\left\{R_{\Gamma_{0}(2), w, w-2 i+1} \mid i=1,2, \ldots, d_{w}\right\}
$$

forms a basis for $S_{w+2}\left(\Gamma_{0}(2)\right)$. 
Now if $f$ is a cusp form of weight $w+2$ on $\Gamma_{0}(2)$ such that $r_{2 i-1}(f)=0$ for all $i=1, \ldots, d_{w}$, then we have $\left(f, R_{\Gamma_{0}(2), w, 2 i-1}\right)=0$ for all $i=1, \ldots, d_{w}$. In view of Theorem 1.4, this implies that $f$ is identically zero. Thus, we see that the map $f \mapsto\left(r_{1}(f), r_{3}(f), \ldots, r_{2 d_{w}-1}(f)\right)$ is an isomorphism and consequently $r^{-}: S_{w+2}\left(\Gamma_{0}(2)\right) \rightarrow V_{w}$ is injective. The same argument applies to other similar maps as well. This is the $\Gamma_{0}(2)$-version of the Eichler-Shimura-Manin theorem ([7], [12, [15], 17]).

Corollary 1.5. The maps

$$
r^{+}, r^{-}: S_{w+2}\left(\Gamma_{0}(2)\right) \rightarrow V_{w}
$$

are both injective.

Note that the $r^{-}$case in Corollary 1.5 was proved in [10] by a different method. However, our result is stronger in the sense that we have actually shown that the function

$$
f \mapsto\left(r_{1}(f), \ldots, r_{2 d_{w}-1}(f)\right)
$$

and other similar functions are isomorphisms between the vector spaces $S_{w+2}\left(\Gamma_{0}(2)\right)$ and $\mathbb{C}^{d_{w}}$.

The proof of Theorem 1.4 can be modified to yield alternative bases for $\Gamma_{0}(2)$, which in turn implies the speculation of Imamoglu and Kohnen [10] mentioned in the introduction that the first $d_{w}$ products $E_{2 j+2}^{i \infty} E_{w-2 j}^{0}, j=1, \ldots, d_{w}$, form a basis.

Theorem 1.6. Let $E_{2 j}^{i \infty}$ and $E_{2 j}^{0}$ be the normalized Eisenstein series of weight $2 j$ on $\Gamma_{0}(2)$ associated with the cusps $i \infty$ and 0 , respectively. Then each of the two sets

$$
\left\{E_{2 j+2}^{0} E_{w-2 j}^{i \infty} \mid j=1, \ldots, d_{w}\right\}, \quad\left\{E_{w-2 j}^{0} E_{2 j+2}^{i \infty} \mid j=1, \ldots, d_{w}\right\}
$$

forms a basis for $S_{w+2}\left(\Gamma_{0}(2)\right)$.

The proof of Theorem 1.6 is slightly more involved than that of Theorem 1.4 since it requires an exact evaluation of Hankel determinants of Bernoulli numbers. The proof of the determinant formulas is supplied by Christian Krattenthaler, and will be given in Appendix A.

Finally, as an application of Theorems 1.1, 1.3 and 1.4, we will give explicit formulas for the Hecke operators on $S_{w+2}\left(\Gamma_{0}(2)\right)$. Let

$$
f(X)=\sum_{\nu=0}^{w} a_{\nu} X^{w-\nu} \quad \text { and } \quad g(X)=\sum_{\nu=0}^{w} b_{\nu} X^{w-\nu}
$$

be polynomials in $X$ with degree $\leq w$. Then their inner product $\langle f, g\rangle$ is defined by

$$
\langle f, g\rangle:=\sum_{\nu=0}^{w} a_{\nu} \bar{b}_{\nu}
$$

where $\bar{b}_{\nu}$ denotes the complex conjugate of $b_{\nu}$.

Under this notation we obtain the following result.

Theorem 1.7. Let $m$ be a positive integer, and let $\mathbf{T}_{m}$ be the matrix representing the Hecke operator

$$
T_{m}: S_{w+2}\left(\Gamma_{0}(2)\right) \rightarrow S_{w+2}\left(\Gamma_{0}(2)\right)
$$

with respect to the basis

$$
c_{w, 2 i} R_{\Gamma_{0}(2), w, 2 i} \quad\left(i=1,2, \ldots, d_{w}\right) .
$$


Let $\mathbf{S}_{1}$ and $\mathbf{S}_{2}$ be matrices defined by

$$
\begin{array}{ll}
\mathbf{S}_{1}:=\left[\left\langle S_{2, w, 2 i}, S_{2, w, 2 j}\right\rangle\right] & \left(i, j=1,2, \ldots, d_{w}\right), \\
\mathbf{S}_{2}:=\left[\left\langle S_{2, w, 2 i}, S_{2, w, 2 j}^{m}\right\rangle\right] & \left(i, j=1,2, \ldots, d_{w}\right) .
\end{array}
$$

Then $\mathbf{T}_{m}$ can be expressed as

$$
\mathbf{T}_{m}=\mathbf{S}_{1}^{-1} \mathbf{S}_{2} .
$$

Consequently, this gives an algorithm for computing the matrix $\mathbf{T}_{m}$ representing the Hecke operator $T_{m}$ on $S_{w+2}\left(\Gamma_{0}(2)\right)$. We append a computer program in the last section.

We conclude this section with two examples.

\section{Examples.}

(1) Consider the Hecke operator $T_{2}$ on $S_{12}\left(\Gamma_{0}(2)\right)$, which is of dimension 2. We have

$$
\begin{aligned}
& S_{2,10,2}(X)=-\frac{1}{45}\left(192 X^{9}-320 X^{7}+168 X^{5}-45 X^{3}+5 X\right), \\
& S_{2,10,4}(X)=\frac{1}{210}\left(160 X^{9}-280 X^{6}+168 X^{4}-55 X^{3}+7 X\right), \\
& S_{2,10,2}^{2}(X)=\frac{128}{45}\left(12 X^{9}+5 X^{7}-42 X^{5}+30 X^{3}-5 X\right), \\
& S_{2,10,4}^{2}(X)=-\frac{32}{105}\left(44 X^{9}-35 X^{7}-42 X^{5}+40 X^{3}-7 X\right) .
\end{aligned}
$$

Thus,

$$
\mathbf{S}_{1}^{-1} \mathbf{S}_{2}=\left(\begin{array}{cc}
-208 & 36 \\
-1120 & 184
\end{array}\right),
$$

whose characteristic polynomial is $x^{2}+24 x+2048$. To check the correctness, we observe that the space $S_{12}\left(\Gamma_{0}(2)\right)$ is spanned by $\Delta(z)$ and $\Delta(2 z)$, where

$$
\Delta(z)=e^{2 \pi i z} \prod_{n=1}^{\infty}\left(1-e^{2 \pi i n z}\right)^{24} .
$$

We find

$$
T_{2} \Delta(z)=-24 \Delta(z)-2048 \Delta(2 z), \quad T_{2} \Delta(2 z)=\Delta(z) .
$$

It is clear that the characteristic polynomial of $T_{2}$ is indeed $x^{2}+24 x+2048$.

(2) Consider $N=4$ and $w=8$. The space $S_{10}\left(\Gamma_{0}(4)\right)$ has dimension 3 with a two-dimensional oldspace coming from $S_{10}\left(\Gamma_{0}(2)\right)$ and a one-dimensional newspace. We can show that $R_{\Gamma_{0}(4), 8,2 i}, i=1,2,3$, form a basis. Thus, by the same reasoning as that for Theorem 1.7, the matrix of a Hecke operator $T_{m}$ with respect to $c_{w, 2 i} R_{\Gamma_{0}(4), 8,2 i}$ would be $\mathbf{S}_{1}^{-1} \mathbf{S}_{2}$, where

$$
\begin{array}{ll}
\mathbf{S}_{1}=\left[\left\langle S_{4,8,2 i}, S_{4,8,2 j}\right\rangle\right] & (i, j=1,2,3), \\
\mathbf{S}_{2}=\left[\left\langle S_{4,8,2 i}, S_{4,8,2 j}^{m}\right\rangle\right] & (i, j=1,2,3) .
\end{array}
$$

Take $m=3$, for example. We find

$$
\mathbf{S}_{1}^{-1} \mathbf{S}_{2}=\frac{1}{152915}\left(\begin{array}{ccc}
2456678965260 & -224610211392 & 61847064000 \\
37961609400000 & -3470759119380 & 955676880000 \\
40281954570000 & -3682878636192 & 1014067309260
\end{array}\right) \text {, }
$$

whose characteristic polynomial is $(x-228)(x+156)^{2}$. Therefore, the eigenvalues of $T_{3}$ of the Hecke eigenform on $\Gamma_{0}(2)$ and the newform on $\Gamma_{0}(4)$ 
of weight 10 are -156 and 228 , respectively. Indeed, the Hecke eigenform on $\Gamma_{0}(2)$ of weight 10 is

$\eta(z)^{8} \eta(2 z)^{8}\left(1-24 \sum_{n=1}^{\infty}\left(\frac{2 n q^{2 n}}{1-q^{2 n}}-\frac{n q^{n}}{1-q^{n}}\right)\right)=q+16 q^{2}-156 q^{3}+256 q^{4}+\cdots$, and the newform on $\Gamma_{0}(4)$ of weight 10 is

$$
\eta(2 z)^{12}\left(1+240 \sum_{n=1}^{\infty} \frac{n^{3} q^{2 n}}{1-q^{2 n}}\right)=q+228 q^{3}-666 q^{5}+\cdots .
$$

\section{Proof of Theorem 1.1}

First we give a characterization of the cusp form $R_{\Gamma_{0}(N), w, n}(z)$ in terms of certain Poincaré series. Recall that $(f, g)$ denotes the Petersson inner product of $f$ and $g$ in $S_{w+2}\left(\Gamma_{0}(N)\right)$. For $\gamma=\left[\begin{array}{ll}a & b \\ c & d\end{array}\right]$ in $G L_{2}^{+}(\mathbb{R}),\left.f\right|_{\gamma}$ is defined by

$$
\left(\left.f\right|_{\gamma}\right)(z):=(\operatorname{det} \gamma)^{w / 2+1}(c z+d)^{-w-2} f\left(\frac{a z+b}{c z+d}\right) .
$$

Lemma 2.1. For $0<n<w$, the cusp form $R_{\Gamma_{0}(N), w, n}$ is given by

$$
R_{\Gamma_{0}(N), w, n}=c_{w, n}^{-1} \sum_{\left[\begin{array}{cc}
a & b \\
c
\end{array}\right] \in \Gamma_{0}(N)} \frac{1}{(a z+b)^{\tilde{n}+1}(c z+d)^{n+1}}, \quad c_{w, n}=(-1)^{n} 2 \pi i\left(\begin{array}{c}
w \\
n
\end{array}\right) .
$$

Proof. The proof for the case $N=1$ is given in [4]. (See also [12.) For general $N$, the lemma is just a special case of Proposition 3 of [1. We reproduce it here for the sake of completeness.

Let $R_{n}$ denote the function on the right-hand side of (2.1). We have

$$
\begin{aligned}
\left(f, c_{w, n} R_{n}\right) & =\left(f,\left.\sum_{\gamma \in \Gamma_{0}(N)} z^{-\tilde{n}-1}\right|_{\gamma}\right)=2 \int_{0}^{\infty} y^{w}\left(\int_{-\infty}^{\infty} \frac{f(x+i y)}{(x-i y)^{\tilde{n}+1}} d x\right) d y \\
& =2 \int_{0}^{\infty} y^{w}\left(\frac{2 \pi i}{\tilde{n} !} f^{(\tilde{n})}(2 i y)\right) d y \\
& =4 \pi i\left(\begin{array}{c}
w \\
n
\end{array}\right)\left(\frac{i}{2}\right)^{\tilde{n}} \int_{0}^{\infty} y^{w-\tilde{n}} f(2 i y) d y \\
& =4 \pi i\left(\begin{array}{c}
w \\
n
\end{array}\right)\left(\frac{i}{2}\right)^{\tilde{n}}(2 i)^{-n-1} r_{n}(f) .
\end{aligned}
$$

Then the lemma follows.

Next we study how $R_{\Gamma_{0}(N), w, n}(z)$ behaves under the Atkin-Lehner involution $\left[\begin{array}{cc}0 & -1 \\ N & 0\end{array}\right]$ :

Lemma 2.2. For all integers $m$ and $n$ with $0 \leq m \leq w$ and $0<n<w$, we have

$$
R_{\Gamma_{0}(N), w, n}\left(\frac{-1}{N z}\right)=(-N)^{\tilde{n}+1} z^{w+2} R_{\Gamma_{0}(N), w, \tilde{n}}(z)
$$

and

$$
r_{m}\left(R_{\Gamma_{0}(N), w, n}\right)=(-N)^{\tilde{n}-m} r_{\tilde{m}}\left(R_{\Gamma_{0}(N), w, \tilde{n}}\right) .
$$


Proof. Recall that the Atkin-Lehner involution $\omega_{N}$ defined by

$$
\left.f\right|_{\omega_{N}}(z)=(\sqrt{N} z)^{-w-2} f\left(\frac{-1}{N z}\right)
$$

is self-adjoint with respect to the Petersson inner product. Thus, we have, for all cusp forms $f$ of weight $w+2$ on $\Gamma_{0}(N)$,

$$
\left(f,\left.R_{\Gamma_{0}(N), w, n}\right|_{\omega_{N}}\right)=\left(\left.f\right|_{\omega_{N}}, R_{\Gamma_{0}(N), w, n}\right)=2(2 i)^{-w-1} \int_{0}^{i \infty}(\sqrt{N} z)^{-w-2} f\left(\frac{-1}{N z}\right) z^{n} d z .
$$

We then make a change of variable $z \mapsto-1 / N z$ in the integral. We find that

$$
\begin{aligned}
\left(f,\left.R_{\Gamma_{0}(N), w, n}\right|_{\omega_{N}}\right) & =2(2 i)^{-w-1} \int_{i \infty}^{0}(\sqrt{N} z)^{w+2} f(z)(-N z)^{-n} \frac{d z}{N z^{2}} \\
& =(-1)^{n+1} N^{w / 2-n}\left(f, R_{\Gamma_{0}(N), w, \tilde{n}}\right) .
\end{aligned}
$$

It follows that

$$
\left.R_{\Gamma_{0}(N), w, n}\right|_{\omega_{N}}=(-1)^{n+1} N^{w / 2-n} R_{\Gamma_{0}(N), w, \tilde{n}},
$$

which is equivalent to our first assertion. We now prove the second assertion.

Since $w_{N}$ is an isometry with respect to the Petersson inner product, we have

$$
\begin{aligned}
2(2 i)^{-w-1} r_{m}\left(R_{\Gamma_{0}(N), w, n}\right) & =\left(R_{\Gamma_{0}(N), w, n}, R_{\Gamma_{0}(N), w, m}\right) \\
& =\left(\left.R_{\Gamma_{0}(N), w, n}\right|_{\omega_{N}},\left.R_{\Gamma_{0}(N), w, m}\right|_{\omega_{N}}\right) .
\end{aligned}
$$

Then by the first part of the lemma, we see that

$$
\begin{aligned}
r_{m}\left(R_{\Gamma_{0}(N), w, n}\right) & =2^{-1}(2 i)^{w+1}(-1)^{m+n} N^{w-n-m}\left(R_{\Gamma_{0}(N), w, \tilde{n}}, R_{\Gamma_{0}(N), w, \tilde{m}}\right) \\
& =(-N)^{\tilde{n}-m} r_{\tilde{m}}\left(R_{\Gamma_{0}(N), w, \tilde{n}}\right) .
\end{aligned}
$$

This completes the proof of the lemma.

Now we are ready to prove the following proposition which is a key for our arguments:

Proposition 2.3. Let $c_{w, n}=(-1)^{n} 2 \pi i\left(\begin{array}{l}w \\ n\end{array}\right)$. For $0 \leq m \leq w$ and $0<n<w$ with opposite parity, we have

(1) if $m+n>w$,

$$
\begin{aligned}
c_{w, n} r_{m}\left(R_{\Gamma_{0}(N), w, n}\right)= & \frac{2 \pi i}{m+1}\left(\begin{array}{c}
m+1 \\
\tilde{n}
\end{array}\right) B_{m-\tilde{n}+1}-\frac{2 \pi i}{N n} \delta_{m, \tilde{n}+1} \\
& \quad-\delta_{m, w}\left(\begin{array}{c}
w+2 \\
n+1
\end{array}\right) \frac{2 \pi i B_{n+1} B_{\tilde{n}+1}}{(w+1) N^{n+1} B_{w+2}} \prod_{p \mid N} \frac{1-p^{-(\tilde{n}+1)}}{1-p^{-(w+2)}}
\end{aligned}
$$

(2) if $m+n<w$,

$$
\begin{aligned}
c_{w, n} r_{m}\left(R_{\Gamma_{0}(N), w, n}\right)=( & -N)^{\tilde{n}-m}\left(\frac{2 \pi i}{\tilde{m}+1}\left(\begin{array}{c}
\tilde{m}+1 \\
n
\end{array}\right) B_{\tilde{m}-n+1}-\frac{2 \pi i}{N \tilde{n}} \delta_{\tilde{m}, n+1}\right. \\
& \left.-\delta_{m, 0}\left(\begin{array}{c}
w+2 \\
n+1
\end{array}\right) \frac{2 \pi i B_{n+1} B_{\tilde{n}+1}}{(w+1) N^{\tilde{n}+1} B_{w+2}} \prod_{p \mid N} \frac{1-p^{-(n+1)}}{1-p^{-(w+2)}}\right),
\end{aligned}
$$

where $\delta_{i, j}$ is the Kronecker delta symbol. 
Furthermore, for an odd integer $m$ with $0<m<w$, we have

$$
\begin{aligned}
r_{m}\left(R_{\Gamma_{0}(N), w, w}\right)= & \frac{B_{m+1}}{m+1}-\frac{\delta_{w, \tilde{m}+1}}{N w} \\
& \quad-\frac{(w+2) B_{m+1} B_{\tilde{m}+1}}{N^{m+1}(m+1)(\tilde{m}+1) B_{w+2}} \prod_{p \mid N} \frac{1-p^{-(\tilde{m}+1)}}{1-p^{-(w+2)}}
\end{aligned}
$$

and

$$
\begin{aligned}
r_{m}\left(R_{\Gamma_{0}(N), w, 0}\right)=- & N^{\tilde{m}}\left(\frac{B_{\tilde{m}+1}}{\tilde{m}+1}-\frac{\delta_{w, m+1}}{N w}\right. \\
& \left.\quad-\frac{(w+2) B_{m+1} B_{\tilde{m}+1}}{N^{\tilde{m}+1}(m+1)(\tilde{m}+1) B_{w+2}} \prod_{p \mid N} \frac{1-p^{-(m+1)}}{1-p^{-(w+2)}}\right) .
\end{aligned}
$$

Proof. To ease the notations, throughout the proof, we write

$$
R_{n}=R_{\Gamma_{0}(N), w, n}=c_{w, n}^{-1} \sum_{\left[\begin{array}{ll}
a & b \\
c & d
\end{array}\right] \in \Gamma_{0}(N)} \frac{1}{(a z+b)^{\tilde{n}+1}(c z+d)^{n+1}} .
$$

Here we first consider the cases where $m>\tilde{n}>0$ (that is, $m+n>w$ ); the results for the remaining cases will follow from these cases using Lemma 2.2. Now for such $m$ and $n$, we partition the sum defining $R_{n}$ into three parts,

$$
c_{w, n} R_{n}(z)=\sum_{c=0}+\sum_{b=0, c \neq 0}+\sum_{b c \neq 0}
$$

and let $S_{i}, i=1,2,3$, denote the $i$-th sum, respectively.

For $S_{1}$ we have

$$
S_{1}(z)=\sum_{\left[\begin{array}{ll}
1 & b \\
0 & 1
\end{array}\right]}+\sum_{\left[\begin{array}{cc}
-1 & -b \\
0 & -1
\end{array}\right]}=2 \sum_{b \in \mathbb{Z}} \frac{1}{(z+b)^{\tilde{n}+1}}=\frac{2(-2 \pi i)^{\tilde{n}+1}}{\tilde{n} !} \sum_{r=1}^{\infty} r^{\tilde{n}} e^{2 \pi i r z},
$$

where in the last equality we use the formula

$$
\sum_{b \in \mathbb{Z}}(z+b)^{-n}=\frac{(-2 \pi i)^{n}}{\Gamma(n)} \sum_{r=1}^{\infty} r^{n-1} e^{2 \pi i r z},
$$

valid for all positive integers $n$ and complex numbers $z$ with $\operatorname{Im} z>0$. It follows that

$$
\begin{aligned}
\int_{0}^{i \infty} z^{m} S_{1}(z) d z & =\frac{2(-2 \pi i)^{\tilde{n}+1}}{\tilde{n} !} \int_{0}^{i \infty} z^{m} \sum_{r=1}^{\infty} r^{\tilde{n}} e^{2 \pi i r z} d z \\
& =2(-2 \pi i)^{\tilde{n}-m} \frac{m !}{\tilde{n} !} \sum_{r=1}^{\infty} r^{-(m-\tilde{n}+1)}=2(-2 \pi i)^{\tilde{n}-m} \frac{m !}{\tilde{n} !} \zeta(m-\tilde{n}+1) .
\end{aligned}
$$

Using the well-known formula

$$
\zeta(2 p)=\frac{(-1)^{p-1}}{(2 p) !} 2^{2 p-1} \pi^{2 p} B_{2 p} \quad(p>0)
$$

we simplify the last expression to

$$
\int_{0}^{i \infty} z^{m} S_{1}(z) d z=\frac{2 \pi i}{m+1}\left(\begin{array}{c}
m+1 \\
\tilde{n}
\end{array}\right) B_{m-\tilde{n}+1}
$$


We next consider the contribution from

$$
S_{2}(z)=2 \sum_{c \in \mathbb{Z}, c \neq 0} \frac{1}{z^{\tilde{n}+1}(c N z+1)^{n+1}} .
$$

We first show that the integral

$$
\int_{0}^{i \infty} z^{m} S_{2}(z) d z
$$

converges absolutely when $m>\tilde{n}+1$. For $z$ with $|z| \ll 1$ we have

$$
\frac{1}{|c N z+1|^{n+1}} \ll \begin{cases}1, & \text { if }|c| \ll 1 /|z|, \\ |c z|^{-n-1}, & \text { if }|c| \gg 1 /|z|,\end{cases}
$$

and hence

$$
\sum_{c \in \mathbb{Z}, c \neq 0} \frac{1}{|c N z+1|^{n+1}} \ll \sum_{|c| \ll 1 /|z|} 1+\frac{1}{|z|^{n+1}} \sum_{|c| \gg 1 /|z|} \frac{1}{|c|^{n+1}} \ll \frac{1}{|z|} .
$$

For $|z| \gg 1$ we have

$$
\frac{1}{|c N z+1|^{n+1}} \ll \frac{1}{|c z|^{n+1}}
$$

and

$$
\sum_{c \in \mathbb{Z}, c \neq 0} \frac{1}{|c N z+1|^{n+1}} \ll \frac{1}{|z|^{n+1}} .
$$

It follows that, for $m>\tilde{n}+1$,

$$
\int_{0}^{i \infty}|z|^{m} \sum_{c \in \mathbb{Z}, c \neq 0} \frac{1}{|z|^{\tilde{n}+1}|c N z+1|^{n+1}} d z \ll \int_{0}^{1} t^{m-\tilde{n}-2} d t+\int_{1}^{\infty} t^{m-\tilde{n}-n-2} d t<\infty .
$$

Now, having proved that $\int_{0}^{i \infty} z^{m} S_{2}(z) d z$ converges absolutely for $m>\tilde{n}+1$, we can change the order of integration and summation freely at will. We have

$$
\begin{aligned}
\int_{0}^{i \infty} z^{m} S_{2}(z) d z & =2 \int_{0}^{i \infty} z^{m-\tilde{n}-1} \sum_{c \in \mathbb{Z}, c \neq 0} \frac{1}{(c N z+1)^{n+1}} d z \\
& =\sum_{c \in \mathbb{Z}, c \neq 0}\left\{\int_{0}^{i \infty} \frac{z^{m-\tilde{n}-1}}{(c N z+1)^{n+1}} d z+\int_{-i \infty}^{0} \frac{(-z)^{m-\tilde{n}-1}}{(-c N z+1)^{n+1}} d z\right\} \\
& =\sum_{c \in \mathbb{Z}, c \neq 0} \int_{-i \infty}^{i \infty} z^{m-\tilde{n}-1} \frac{1}{(c N z+1)^{n+1}} d z .
\end{aligned}
$$

We then move the line of integration to the far left or the far right according to the sign of $c$. Thus, the integral is 0 , provided that $m>\tilde{n}+1$.

For the case $m=\tilde{n}+1$, we first observe that, for large $z$, we have (2.5), which in term implies that

$$
\int_{i \epsilon}^{i \infty} z^{m} S_{2}(z) d z
$$


converges absolutely for any given $\epsilon>0$. It follows that

$$
\begin{aligned}
\int_{0}^{\infty} z^{m} S_{2}(z) d z & =2 \lim _{\epsilon \rightarrow 0} \int_{i \epsilon}^{i \infty} \sum_{c \in \mathbb{Z}, c \neq 0} \frac{d z}{(c N z+1)^{n+1}} \\
& =2 \lim _{\epsilon \rightarrow 0} \sum_{c \in \mathbb{Z}, c \neq 0} \int_{i \epsilon}^{i \infty} \frac{d z}{(c N z+1)^{n+1}}=\lim _{\epsilon \rightarrow 0} \frac{2}{N n} \sum_{c \in \mathbb{Z}, c \neq 0} \frac{1}{c(i c N \epsilon+1)^{n}} \\
& =\lim _{\epsilon \rightarrow 0} \frac{2 \epsilon}{N n} \sum_{c=1}^{\infty} \frac{(1-i c N \epsilon)^{n}-(1+i c N \epsilon)^{n}}{(c \epsilon)\left(1+c^{2} N^{2} \epsilon^{2}\right)^{n}} .
\end{aligned}
$$

Now the last expression is a Riemann sum of the integral

$$
2 \int_{0}^{\infty} \frac{(1-i N x)^{n}-(1+i N x)^{n}}{N n x\left(1+N^{2} x^{2}\right)^{n}} d x=\int_{-\infty}^{\infty} \frac{(1-i N x)^{n}-(1+i N x)^{n}}{N n x\left(1+N^{2} x^{2}\right)^{n}} d x
$$

which, by moving the line of integration to $\operatorname{Im} x=\infty$ and counting the residue at $i / N$, is shown to be equal to $-2 \pi i /(N n)$. Thus, we conclude that

$$
\int_{0}^{i \infty} z^{m} S_{2}(z) d z= \begin{cases}0, & \text { if } m>\tilde{n}+1 \\ -2 \pi i /(N n), & \text { if } m=\tilde{n}+1\end{cases}
$$

We now consider the contribution from $S_{3}(z)$. For this it is necessary to distinguish the two cases $m<w$ and $m=w$. For the former case $m<w$, again, we first show that the integral

$$
\int_{0}^{i \infty} z^{m} S_{3}(z) d z=\int_{0}^{i \infty} z^{m} \sum_{b c \neq 0} \frac{d z}{(a z+b)^{\tilde{n}+1}(c z+d)^{n+1}}
$$

converges absolutely. For $|z| \ll 1$, write

$$
S_{3}(z)=\sum_{b, d} \frac{1}{b^{\tilde{n}+1} d^{n+1}} \sum_{h}\left(\frac{c z}{d}+\frac{z}{b d}+h N z+1\right)^{-(\tilde{n}+1)}\left(\frac{c}{d} z+h N z+1\right)^{-(n+1)}
$$

where in the inner sum, for a given pair of $b$ and $d$, the number $c$ is an integer satisfying $N|c| c \mid, \leq d N$, and $b c \equiv-1 \bmod d$, and $h$ runs over all integers satisfying $c / d+h N \neq 0$. (In particular, when $d \neq \pm 1, h$ runs over all integers.) Now

$$
\begin{aligned}
\mid \frac{c z}{d}+ & \frac{z}{b d}+h N z+\left.1\right|^{-(\tilde{n}+1)}\left|\frac{c}{d} z+h N z+1\right|^{-(n+1)} \\
& \ll \begin{cases}1, & \text { if }|h N+c / d| \ll 1 /|z|, \\
|(h N+c / d) z|^{-w-2}, & \text { if }|h N+c / d| \gg 1 /|z| .\end{cases}
\end{aligned}
$$

It follows that

$$
\sum_{\left[\begin{array}{ll}
a & b \\
c & d
\end{array}\right] \in \Gamma_{0}(N), b c \neq 0} \frac{1}{|a z+b|^{\tilde{n}+1}|c z+d|^{n+1}} \ll \frac{1}{|z|}
$$


for $|z| \ll 1$. Furthermore, for $|z| \gg 1$, we write

$$
\begin{aligned}
& \quad \sum_{\left[\begin{array}{ll}
a & b \\
c & d
\end{array}\right]} \frac{1}{|a z+b|_{0}(N), b c \neq 0} \frac{1}{|c+1| c z+\left.d\right|^{n+1}} \\
& \quad=\frac{1}{N^{n+1}|z|^{w+2}} \sum_{\left[\begin{array}{cc}
d & -c / N \\
-b N & a
\end{array}\right] \in \Gamma_{0}(N), b c \neq 0} \frac{1}{|a+b N /(N z)|^{\tilde{n}+1}|c / N+d /(N z)|^{n+1}} .
\end{aligned}
$$

Then by the same argument as before, we find that the sum is bounded by $|z|^{-w-1}$. It follows that

$$
\int_{0}^{i \infty} z^{m} S_{3}(z) d z
$$

converges absolutely when $m<w$ and we may change the order of integration and summation freely. Now we have

$$
\begin{aligned}
S_{3}(-z) & =\sum_{\left[\begin{array}{ll}
a & b \\
c & d
\end{array}\right] \in \Gamma_{0}(N), b c \neq 0} \frac{1}{(-a z+b)^{\tilde{n}+1}(-c z+d)^{n+1}} \\
& =(-1)^{\tilde{n}+1} \sum_{\left[\begin{array}{ll}
a & b \\
c & d
\end{array}\right] \in \Gamma_{0}(N), b c \neq 0} \frac{1}{(a z-b)^{\tilde{n}+1}(-c z+d)^{n+1}}=(-1)^{\tilde{n}+1} S_{3}(z)
\end{aligned}
$$

and thus

$$
\int_{0}^{i \infty} z^{m} S_{3}(z) d z=\frac{1}{2} \int_{-i \infty}^{i \infty} z^{m} S_{3}(z) d z
$$

Furthermore, for each $\left[\begin{array}{ll}a & b \\ c & d\end{array}\right] \in \Gamma_{0}(N)$ with $b c \neq 0$ we have $a b c d>0$ and consequently the two poles $-b / a$ and $-d / c$ of $z^{m} /(a z+b)^{\tilde{n}+1}(c z+d)^{n+1}$ lie on the same side of the imaginary axis. This implies that

$$
\int_{-i \infty}^{i \infty} \frac{z^{m}}{(a z+b)^{\tilde{n}+1}(c z+d)^{n+1}} d z=0
$$

for all $\left[\begin{array}{ll}a & b \\ c & d\end{array}\right] \in \Gamma_{0}(N)$ with $b c \neq 0$ and we have

$$
\int_{0}^{i \infty} z^{m} S_{3}(z) d z=0
$$

when $m<w$.

We now consider the contribution of $S_{3}$ for the case $m=w$. We write, by (2.9),

$$
\begin{aligned}
\int_{0}^{i \infty} z^{w} S_{3}(z) d z & =\frac{1}{2} \lim _{\epsilon \rightarrow 0} \int_{-i / \epsilon}^{i / \epsilon} z^{w} S_{3}(z) d z \\
& =\frac{1}{2} \lim _{\epsilon \rightarrow 0} \sum_{\left[\begin{array}{ll}
a & b \\
c & d
\end{array}\right] \in \Gamma_{0}(N), b c \neq 0} \int_{-i / \epsilon}^{i / \epsilon} \frac{z^{w} d z}{(a z+b)^{\tilde{n}+1}(c z+d)^{n+1}} .
\end{aligned}
$$

Here the change of order of summation and integration is justified by the estimate (2.8). Using (2.10) we reduce the last expression to

$$
-\frac{1}{2} \lim _{\epsilon \rightarrow 0} \sum_{\left[\begin{array}{ll}
a & b \\
c & d
\end{array}\right] \in \Gamma_{0}(N), b c \neq 0}\left(\int_{i / \epsilon}^{i \infty}+\int_{-i \infty}^{-i / \epsilon}\right) \frac{z^{w} d z}{(a z+b)^{\tilde{n}+1}(c z+d)^{n+1}} .
$$


We then make a change of variable $z=i /(\epsilon t)$ and obtain

$$
\int_{0}^{i \infty} z^{w} S_{3}(z) d z=-\lim _{\epsilon \rightarrow 0} \sum_{\left[\begin{array}{ll}
a & b \\
c & d
\end{array}\right] \in \Gamma_{0}(N), b c \neq 0} \frac{\epsilon}{2 i} \int_{-1}^{1} \frac{d t}{(a-i \epsilon t b)^{\tilde{n}+1}(c-i \epsilon t d)^{n+1}} .
$$

We rewrite the sum as

$$
\sum_{\left[\begin{array}{ll}
a & b \\
c & d
\end{array}\right] \in \Gamma_{0}(N), c \neq 0}-\sum_{\left[\begin{array}{ll}
1 & 0 \\
c & 1
\end{array}\right] \in \Gamma_{0}(N), c \neq 0}-\sum_{\left[\begin{array}{cc}
-1 & 0 \\
c & -1
\end{array}\right] \in \Gamma_{0}(N), c \neq 0} .
$$

The latter two sums are bounded above by

$$
\ll \epsilon \sum_{c=1}^{\infty} \int_{-1}^{1} \frac{d t}{|c-i \epsilon t|^{n+1}} \ll \epsilon \sum_{c=1}^{\infty} \frac{1}{c^{n+1}} \ll \epsilon .
$$

Thus, as $\epsilon$ tends to 0 , the latter two sums vanish. Consequently we have

$$
\begin{aligned}
\int_{0}^{i \infty} z^{w} S_{3}(z) d z=- & \lim _{\epsilon \rightarrow 0} \sum_{\left[\begin{array}{ll}
a & b \\
c & d
\end{array}\right] \in \Gamma_{0}(N), c \neq 0} \frac{\epsilon}{2 i} \int_{-1}^{1} \frac{d t}{(a-i \epsilon t b)^{\tilde{n}+1}(c-i \epsilon t d)^{n+1}} \\
=- & \lim _{\epsilon \rightarrow 0} \sum_{(a, c)=1, N \mid c, c \neq 0} \frac{1}{a^{\tilde{n}+1} c^{n+1}} \\
& \times \sum_{h \in \mathbb{Z}+b / a} \frac{\epsilon}{2 i} \int_{-1}^{1} \frac{d t}{(1-i \epsilon t h)^{\tilde{n}+1}(1-i \epsilon t(h+1 / a c))^{n+1}},
\end{aligned}
$$

where for a given pair $(a, c), b$ denotes an integer satisfying $b c \equiv-1 \bmod a$. Now the inner sum is a Riemann sum of

$$
\frac{1}{2 i} \int_{-\infty}^{\infty} \int_{-1}^{1} \frac{d t}{(1-i x t)^{w+2}} d x
$$

The evaluation of this integral is similar to that of (2.6) and we find that it equals

$$
-\frac{1}{2(w+1)} \int_{-\infty}^{\infty} \frac{1}{x}\left(\frac{1}{(1-i x)^{w+1}}-\frac{1}{(1+i x)^{w+1}}\right) d x=-\frac{\pi i}{w+1} .
$$

Therefore, we have

$$
\int_{0}^{i \infty} z^{w} S_{3}(z) d z=\frac{\pi i}{w+1} \sum_{(a, c)=1, N \mid c, c \neq 0} \frac{1}{a^{\tilde{n}+1} c^{n+1}} .
$$

Finally, we find

$$
\begin{aligned}
\sum_{(a, c)=1, N \mid c, c \neq 0} \frac{1}{a^{\tilde{n}+1} c^{n+1}} & =4 \sum_{a=1,(a, N)=1}^{\infty} \frac{1}{a^{\tilde{n}+1}} \sum_{c=1,(a, c)=1}^{\infty} \frac{1}{(c N)^{n+1}} \\
& =\frac{4}{N^{n+1}} \sum_{a=1,(a, N)=1}^{\infty} \frac{1}{a^{\tilde{n}+1}} \sum_{d \mid a} \mu(d) \sum_{c=1}^{\infty} \frac{1}{(c d)^{n+1}} \\
& =\frac{4 \zeta(n+1)}{N^{n+1}} \sum_{d=1,(d, N)=1}^{\infty} \frac{\mu(d)}{d^{n+1}} \sum_{a=1,(a, N)=1}^{\infty} \frac{1}{(a d)^{\tilde{n}+1}} \\
& =\frac{4 \zeta(n+1) \zeta(\tilde{n}+1)}{\zeta(w+2) N^{n+1}} \prod_{p \mid N} \frac{1-p^{-(\tilde{n}+1)}}{1-p^{-(w+2)}},
\end{aligned}
$$


where the product is taking over all prime divisors $p$ of $N$. With (2.3) the last expression becomes

$$
-\frac{2}{N^{n+1}}\left(\begin{array}{c}
w+2 \\
n+1
\end{array}\right) \frac{B_{n+1} B_{\tilde{n}+1}}{B_{w+2}} \prod_{p \mid N} \frac{1-p^{-(\tilde{n}+1)}}{1-p^{-(w+2)}} .
$$

In summary, we see that, for integers $m$ and $n, 0 \leq m \leq w, 0<n<w$, with opposite parity and $m+n>w$,

$$
\int_{0}^{i \infty} z^{m} S_{3}(z) d z= \begin{cases}0, & \text { if } m<w \\
-\left(\begin{array}{c}
w+2 \\
n+1
\end{array}\right) \frac{2 \pi i B_{n+1} B_{\tilde{n}+1}}{(w+1) N^{n+1} B_{w+2}} \prod_{p \mid N} \frac{1-p^{-(\tilde{n}+1)}}{1-p^{-(w+2)}}, & \text { if } m=w .\end{cases}
$$

Combining (2.4), (2.7) and (2.12), we conclude that

$$
\begin{aligned}
c_{w, n} r_{m}\left(R_{\Gamma_{0}(N), w, n}\right)= & \frac{2 \pi i}{m+1}\left(\begin{array}{c}
m+1 \\
\tilde{n}
\end{array}\right) B_{m-\tilde{n}+1}-\frac{2 \pi i}{N n} \delta_{m, \tilde{n}+1} \\
& \quad-\delta_{m, w}\left(\begin{array}{c}
w+2 \\
n+1
\end{array}\right) \frac{2 \pi i B_{n+1} B_{\tilde{n}+1}}{(w+1) N^{n+1} B_{w+2}} \prod_{p \mid N} \frac{1-p^{-(\tilde{n}+1)}}{1-p^{-(w+2)}}
\end{aligned}
$$

for the cases $m+n>w$.

For integers $m$ and $n$ satisfying $m \not \equiv n \bmod 2$ and $m<\tilde{n}$, or equivalently, $m+n<w$, we make use of Lemma 2.2. We have

$$
r_{m}\left(R_{n}\right)=(-N)^{\tilde{n}-m} r_{\tilde{m}}\left(R_{\tilde{n}}\right) .
$$

Now $\tilde{m}+\tilde{n}>w$ and we can apply the formula just obtained with $m$ and $n$ replaced by $\tilde{m}$ and $\tilde{n}$, respectively. We find that

$$
\begin{aligned}
c_{w, n} r_{m}\left(R_{n}\right)=(-N)^{\tilde{n}-m}\left(\frac{2 \pi i}{\tilde{m}+1}\left(\begin{array}{c}
\tilde{m}+1 \\
n
\end{array}\right) B_{\tilde{m}-n+1}-\frac{2 \pi i}{N \tilde{n}} \delta_{\tilde{m}, n+1}\right. \\
\left.\quad-\delta_{m, 0}\left(\begin{array}{c}
w+2 \\
n+1
\end{array}\right) \frac{2 \pi i B_{n+1} B_{\tilde{n}+1}}{(w+1) N^{\tilde{n}+1} B_{w+2}} \prod_{p \mid N} \frac{1-p^{-(n+1)}}{1-p^{-(w+2)}}\right) .
\end{aligned}
$$

This proves the the proposition for the cases $0<n<w$. Now for the cases $n=0$ and $n=w$, by Definition 1.1, we have

$$
r_{m}\left(R_{0}\right)=2^{-1}(2 i)^{w+1}\left(R_{0}, R_{m}\right)=2^{-1}(2 i)^{w+1} \overline{\left(R_{m}, R_{0}\right)}=-\overline{r_{0}\left(R_{m}\right)}
$$

and also

$$
r_{m}\left(R_{w}\right)=-\overline{r_{w}\left(R_{m}\right)}
$$

At this point we insert the formulas just obtained into the equations above. After simplification, we arrive at the claimed formulas. This completes the proof.

We now give a proof of Theorem 1.1 . 
Proof of Theorem 1.1. By Proposition 2.3, we have

$$
\begin{aligned}
r^{-}\left(R_{\Gamma_{0}(N), w, n}\right)(X)= & -\sum_{\substack{0<m<w \\
m \text { odd }}}\left(\begin{array}{c}
w \\
m
\end{array}\right) X^{m} r_{\tilde{m}}\left(R_{\Gamma_{0}(N), w, n}\right) \\
= & -\left(\begin{array}{c}
w \\
n
\end{array}\right)^{-1} \sum_{\substack{0<m<n \\
m \text { odd }}}\left(\begin{array}{c}
w \\
m
\end{array}\right)\left(\begin{array}{c}
\tilde{m}+1 \\
\tilde{n}
\end{array}\right) \frac{X^{m}}{\tilde{m}+1} B_{\tilde{m}-\tilde{n}+1} \\
& +\left(\begin{array}{c}
w \\
n
\end{array}\right)^{-1} \sum_{\substack{n<m<w \\
m \text { odd }}}\left(\begin{array}{c}
w \\
m
\end{array}\right)\left(\begin{array}{c}
m+1 \\
n
\end{array}\right) \frac{N^{\tilde{n}-\tilde{m}} X^{m}}{m+1} B_{m-n+1} \\
& +\left(\begin{array}{c}
w \\
n
\end{array}\right)^{-1}\left(\begin{array}{c}
w \\
n-1
\end{array}\right) \frac{X^{n-1}}{N n}-\left(\begin{array}{c}
w \\
n
\end{array}\right)^{-1}\left(\begin{array}{c}
w \\
n+1
\end{array}\right) \frac{X^{n+1}}{\tilde{n}} .
\end{aligned}
$$

After a straightforward simplification, the first sum in the last expression becomes

$$
\frac{1}{n+1} \sum_{\substack{0<m<n \\
m \text { odd }}}\left(\begin{array}{c}
n+1 \\
m
\end{array}\right) X^{m} B_{n-m+1}=\frac{1}{n+1}\left(B_{n+1}^{0}(X)-X^{n+1}\right),
$$

while the second sum is reduced to

$$
\begin{aligned}
\frac{1}{\tilde{n}+1} \sum_{\substack{n<m<w \\
m \text { odd }}}\left(\begin{array}{c}
\tilde{n}+1 \\
\tilde{m}
\end{array}\right) N^{\tilde{n}-\tilde{m}} X^{w-\tilde{m}} B_{\tilde{n}-\tilde{m}+1} \\
\quad=\frac{N^{\tilde{n}} X^{w}}{\tilde{n}+1}\left\{B_{\tilde{n}+1}^{0}\left(\frac{1}{N X}\right)-\frac{1}{(N X)^{\tilde{n}+1}}\right\} .
\end{aligned}
$$

Altogether, we see that

$$
r^{-}\left(R_{\Gamma_{0}(N), w, n}\right)(X)=\frac{N^{\tilde{n}} X^{w}}{\tilde{n}+1} B_{\tilde{n}+1}^{0}\left(\frac{1}{N X}\right)-\frac{1}{n+1} B_{n+1}^{0}(X),
$$

as claimed in the statement.

The proof of the statement about $r^{+}\left(R_{\Gamma_{0}(N), w, n}\right)$ for odd $n$ is almost the same, except that there are two extra terms

$$
c_{w, n}^{-1}\left(\begin{array}{c}
w+2 \\
n+1
\end{array}\right) \frac{2 \pi i B_{n+1} B_{\tilde{n}+1}}{(w+1) B_{w+2}}\left(\frac{1}{N^{n+1}} \prod_{p \mid N} \frac{1-p^{-(\tilde{n}+1)}}{1-p^{-(w+2)}}-\frac{X^{w}}{N} \prod_{p \mid N} \frac{1-p^{-(n+1)}}{1-p^{-(w+2)}}\right) .
$$

We shall omit the details here.

\section{Proof of Lemma 1.2 and Theorem 1.3}

Let $m$ be a positive integer. Recall that the congruence subgroup $\Gamma_{0}(N)$ acts on the set

$H_{N, m}:=\left\{\left[\begin{array}{ll}a & b \\ c & d\end{array}\right] \mid a, b, c, d \in \mathbb{Z} ; a d-b c=m ; c \equiv 0 \quad(\bmod N) ; \operatorname{gcd}(a, N)=1\right\}$

by matrix multiplication on the left. The standard set of coset representatives is given by

$$
M_{N, m}:=\left\{\left[\begin{array}{ll}
a & b \\
0 & d
\end{array}\right] \mid a, d \in \mathbb{Z}^{+} ; a d=m ; \operatorname{gcd}(a, N)=1 ; b=0, \ldots, d-1 ;\right\} .
$$

In particular, we have the following lemma. 
Lemma 3.1. One has

$$
\Gamma_{0}(N) M_{N, m}=H_{N, m} .
$$

With this lemma, it is straightforward to prove Lemma 1.2

Proof of Lemma 1.2. Let $f \in S_{w+2}\left(\Gamma_{0}(N)\right)$ be a cusp form. Recall that the Hecke operator $T_{m}$ is defined by

$$
T_{m} f(z)=m^{w / 2} \sum_{\alpha \in M_{N, m}}\left(\left.f\right|_{\alpha}\right)(z) .
$$

Thus, from Lemma 2.1, we have

$$
\begin{aligned}
T_{m} R_{\Gamma_{0}(N), w, n}(z) & =c_{w, n}^{-1} T_{m} \sum_{\left[\begin{array}{ll}
a & b \\
c & d
\end{array}\right] \in \Gamma_{0}(N)} \frac{1}{(a z+b)^{\tilde{n}+1}(c z+d)^{n+1}} \\
& =\left.c_{w, n}^{-1} T_{m} \sum_{\gamma \in \Gamma_{0}(N)} z^{-\tilde{n}-1}\right|_{\gamma} \\
& =\left.\left.m^{w / 2} c_{w, n}^{-1} \sum_{\alpha \in M_{N, m}} \sum_{\gamma \in \Gamma_{0}(N)} z^{-\tilde{n}-1}\right|_{\gamma}\right|_{\alpha} \\
& =\left.m^{w / 2} c_{w, n}^{-1} \sum_{\gamma^{\prime} \in H_{N, m}} z^{-\tilde{n}-1}\right|_{\gamma^{\prime}} \\
& =m^{w+1} c_{w, n}^{-1} \sum_{\left[\begin{array}{c}
a^{\prime} \\
c^{\prime} \\
d^{\prime}
\end{array}\right] \in H_{N, m}} \frac{1}{\left(a^{\prime} z+b^{\prime}\right)^{\tilde{n}+1}\left(c^{\prime} z+d^{\prime}\right)^{n+1}} \\
& =R_{\Gamma_{0}(N), w, n}^{m}(z) .
\end{aligned}
$$

Here we used the identity

$$
H_{N, m}=\Gamma_{0}(N) M_{N, m}
$$

in Lemma 3.1. This completes the proof.

Proof of Theorem 1.3. The proofs of Proposition 2.3 and Theorem 1.1 are valid for this theorem with a few modifications. Let $n$ be an even integer and write

$$
R_{n}^{m}(z)=R_{\Gamma_{0}(N), w, n}^{m}=\frac{m^{w+1}}{c_{w, n}} \sum_{\left[\begin{array}{ll}
a & b \\
c & d
\end{array}\right] \in H_{N, m}} \frac{1}{(a z+b)^{\tilde{n}+1}(c z+d)^{n+1}} .
$$

We are required to evaluate the integrals

$$
\int_{0}^{i \infty} z^{j} R_{n}^{m}(z) d z
$$

for odd integers $j$.

For $j$ with $j>\tilde{n}$, we partition the $\operatorname{sum} R_{n}^{m}(z)$ as

$m^{-w-1} c_{w, n} R_{n}^{m}(z)=\sum_{c=0}+\sum_{b=0, c \neq 0}+\sum_{a b c d>0}+\sum_{d=0}+\sum_{a b c d<0}=S_{1}+S_{2}+S_{3}+S_{4}+S_{5}$.

The evaluation of $\int_{0}^{i \infty} S_{i}(z) z^{j} d z, i=1,2,3$, is almost the same as that of the corresponding integrals in Proposition 2.3. For instance, if we replace $z$ by $a z$ in 
(2.2), multiply by $d^{-(n+1)}$ and sum over all pairs of $(a, d)$ satisfying $a d=m$, then we get the $S_{1}(z)$ function here. Thus, it is easy to see that

$$
\int_{0}^{i \infty} z^{j} S_{1}(z) d z=\frac{2 \pi i}{j+1}\left(\begin{array}{c}
j+1 \\
\tilde{n}
\end{array}\right) B_{j-\tilde{n}+1} \sum_{\substack{a d=m, a>0 \\
\operatorname{gcd}(a, N)=1}} \frac{1}{a^{j+1} d^{n+1}}
$$

for $j>\tilde{n}$. Likewise, for $S_{2}(z)$ we have

$$
\int_{0}^{i \infty} z^{j} S_{2}(z) d z= \begin{cases}0, & \text { if } j>\tilde{n}+1, \\ -\frac{2 \pi i}{N n} \sum_{\substack{a d=m, a>0 \\ \operatorname{gcd}(a, N)=1}} \frac{1}{a^{\tilde{n}+1} d^{n}}, & \text { if } j=\tilde{n}+1 .\end{cases}
$$

Furthermore, the argument in Proposition 2.3 shows that

$$
\int_{0}^{i \infty} z^{j} S_{3}(z) d z=0
$$

since $j$ is assumed to be odd and can not be 0 or $w$.

The sum $S_{4}$ is actually empty unless $N \mid m$. When this occurs, we have

$$
\begin{aligned}
& S_{4}(z)=\sum_{\left[\begin{array}{cc}
a & b \\
c N & 0
\end{array}\right] \in H_{N, m}} \frac{1}{(a z+b)^{\tilde{n}+1}(c N z)^{n+1}} \\
& =2 \sum_{\substack{c>0,-b c N=m}} \frac{1}{(c N z)^{n+1}} \sum_{\substack{h=1, \operatorname{gcd}(h, N)=1}}^{N} \sum_{a \in \mathbb{Z}} \frac{1}{((a N+h) z+b)^{\tilde{n}+1}} .
\end{aligned}
$$

The innermost sum is equal to

$$
\frac{1}{(N z)^{\tilde{n}+1}} \sum_{a \in \mathbb{Z}} \frac{1}{(a+h / N+b / N z)^{\tilde{n}+1}}=\frac{(-2 \pi i)^{\tilde{n}+1}}{(N z)^{\tilde{n}+1} \tilde{n} !} \sum_{r=1}^{\infty} r^{\tilde{n}} e^{2 \pi i r(h / N+b / N z)} .
$$

Set

$$
\begin{aligned}
f(r) & =\sum_{\substack{h=1 \\
\operatorname{gcd}(h, N)=1}}^{N} e^{2 \pi i r h / N}=\sum_{d \mid N} \mu(d) \sum_{h=1}^{N / d} e^{2 \pi i r h d / N} \\
& =N \sum_{d|N, N / d| r} \frac{\mu(d)}{d}=\sum_{d \mid \operatorname{gcd}(N, r)} \mu(N / d) d .
\end{aligned}
$$

Then we have

$$
\begin{aligned}
\int_{0}^{i \infty} z^{j} S_{4}(z) d z & =\frac{2(-2 \pi i)^{\tilde{n}+1}}{N^{w+2} \tilde{n} !} \int_{0}^{i \infty} z^{-(\tilde{j}+2)} \sum_{\substack{c>0 \\
-b c N=m}} \frac{1}{c^{n+1}} \sum_{r=1}^{\infty} f(r) r^{\tilde{n}} e^{2 \pi i r b / N z} d z \\
& =\frac{2(-2 \pi i)^{\tilde{n}+1}}{N^{w+2} \tilde{n} !} \int_{0}^{i \infty} z^{\tilde{j}} \sum_{\substack{c>0 \\
-b c N=m}} \frac{1}{c^{n+1}} \sum_{r=1}^{\infty} f(r) r^{\tilde{n}} e^{2 \pi i r|b| z / N} d z,
\end{aligned}
$$

where $\tilde{j}=w-j$. When $n>j$, we may integrate term by term, and the result is

$$
\int_{0}^{i \infty} z^{j} S_{4}(z) d z=\frac{2(-2 \pi i)^{j-n}}{N^{j+1}} \frac{\tilde{j} !}{\tilde{n} !} \sum_{\substack{c>0 \\-b c N=m}} \frac{1}{c^{n+1}|b|^{\tilde{j}+1}} \sum_{r=1}^{\infty} \frac{f(r)}{r^{n-j+1}} .
$$


Now we have

$$
\sum_{r=1}^{\infty} \frac{f(r)}{r^{n-j+1}}=\sum_{d \mid N} \mu(N / d) d \sum_{r=1}^{\infty} \frac{1}{(d r)^{n-j+1}}=\zeta(n-j+1) \sum_{d \mid N} \mu(N / d) d^{j-n} .
$$

Simplifying the result, we get

$$
\begin{aligned}
\int_{0}^{i \infty} z^{j} S_{4}(z) d z & =\frac{2 \pi i B_{n-j+1} \tilde{j} !}{N^{j+1}(n-j+1) ! \tilde{n} !} \sum_{\substack{c>0 \\
-b c N=m}} \frac{1}{c^{n+1}|b|^{\tilde{j}+1}} \sum_{d \mid N} \mu(N / d) d^{j-n} \\
& =\frac{2 \pi i N^{w-2 j} B_{n-j+1}}{(\tilde{j}+1) m^{\tilde{j}+1}}\left(\begin{array}{c}
\tilde{j}+1 \\
\tilde{n}
\end{array}\right) \sum_{c \mid(m / N)} c^{\tilde{j}-n} \sum_{d \mid N} \mu(N / d) d^{j-n}
\end{aligned}
$$

for $n>j$.

For $n<j$, we write

$$
\int_{0}^{i \infty} z^{j} S_{4}(z) d z=i^{j+1} \int_{0}^{\infty} t^{j} S_{4}(i t) d t
$$

and observe that the integral

$$
\int_{0}^{\infty} t^{s} S_{4}(i t) d t
$$

defines an analytic function for $\operatorname{Re} s<n$. Thus, by the uniqueness of analytic continuation, the formula

$$
\int_{0}^{i \infty} z^{j} S_{4}(z) d z=\frac{2(-2 \pi i)^{j-n}}{N^{j+1}} \frac{\tilde{j} !}{\tilde{n} !} \zeta(n-j+1) \sum_{\substack{c>0 \\-b c N=m}} \frac{1}{c^{n+1}|b|^{\tilde{j}+1}} \sum_{d \mid N} \mu(N / d) d^{j-n} .
$$

remains valid for $n<j$. In summary, we have shown that

$$
\int_{0}^{i \infty} z^{j} S_{4}(z) d z=\frac{2 \pi i N^{w-2 j} B_{n-j+1}}{(\tilde{j}+1) m^{\tilde{j}+1}}\left(\begin{array}{c}
\tilde{j}+1 \\
\tilde{n}
\end{array}\right) \sum_{c \mid(m / N)} c^{\tilde{j}-n} \sum_{d \mid N} \mu(N / d) d^{j-n}
$$

where $B_{\ell}$ is understood to be 0 if $\ell<0$.

For $S_{5}$, we have

$$
\sum_{a b c d<0} \int_{0}^{i \infty} \frac{z^{j}}{(a z+b)^{\tilde{n}+1}(c z+d)^{n+1}} d z=\frac{1}{2} \sum_{a b c d<0} \int_{-i \infty}^{i \infty} \frac{z^{j}}{(a z+b)^{\tilde{n}+1}(c z+d)^{n+1}} d z .
$$

To evaluate the integrals, it turns out to be easier to work with the polynomial

$$
F(X)=\int_{-i \infty}^{i \infty} \frac{(X-z)^{w}}{(a z+b)^{\tilde{n}+1}(c z+d)^{n+1}} d z
$$

instead. Observe that

for $\ell=0,1, \ldots, n-1$, and

$$
\left.\frac{d^{\ell}}{d X^{\ell}} F(X)\right|_{X=-b / a}=0
$$

$$
\left.\frac{d^{\ell}}{d X^{\ell}} F(X)\right|_{X=-d / c}=0
$$

for $\ell=0,1 \ldots, \tilde{n}-1$. This is because in these cases the integrands have only one pole at $-d / c$ for the former cases and at $-b / a$ for the latter cases. This implies that

$$
F(X)=C(a X+b)^{n}(c X+d)^{\tilde{n}}
$$


for some constant $C$. To determine the constant $C$, we consider

$$
\left.\frac{d^{n}}{d X^{n}} F(X)\right|_{X=-b / a}
$$

Using the integral definition of $F(X)$, we have

$$
\begin{aligned}
\left.\frac{d^{n}}{d X^{n}} F(X)\right|_{X=-b / a} & =a^{-\tilde{n}} \frac{w !}{\tilde{n} !} \int_{-i \infty}^{i \infty} \frac{d z}{(a z+b)(c z+d)^{n+1}} \\
& =2 \pi i \operatorname{sgn}(a b) a^{-\tilde{n}-1} \frac{w !}{\tilde{n} !}(-b c / a+d)^{-n-1} \\
& =2 \pi i \operatorname{sgn}(a b) m^{-n-1} a^{n-\tilde{n}} \frac{w !}{\tilde{n} !}
\end{aligned}
$$

On the other hand, using (3.5) we have

$$
\left.\frac{d^{n}}{d X^{n}} F(X)\right|_{X=-b / a}=C n ! a^{n}(-b c / a+d)^{\tilde{n}}=C m^{\tilde{n}} a^{n-\tilde{n}} n ! .
$$

Comparing the two expressions, we see that $C=m^{-w-1} \operatorname{sgn}(a b) c_{w, n}$ and

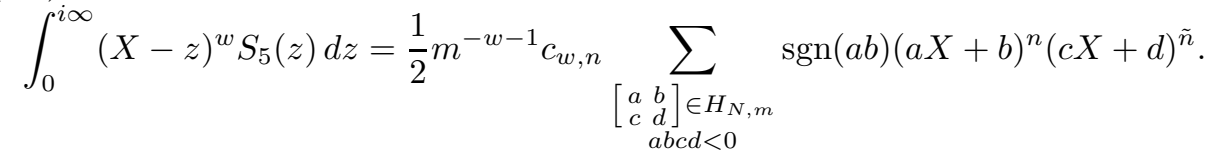

Altogether, (3.1), (3.2), (3.3), (3.4), and (3.6) give the evaluation of $r_{j}\left(R_{\Gamma_{0}(N), w, n}^{m}\right)$ for $j>\tilde{n}$.

For the cases $j<\tilde{n}$, we partition the sum

$$
m^{-w-1} c_{w, n} R_{\Gamma_{0}(N), w, n}^{m}(z)
$$

as

$$
\sum_{b=0}+\sum_{c=0, b \neq 0}+\sum_{a b c d>0}+\sum_{d=0}+\sum_{a b c d<0}=S_{1}+S_{2}+S_{3}+S_{4}+S_{5}
$$

instead. The terms $S_{3}, S_{4}$, and $S_{5}$ are the same as before. For $S_{1}$ and $S_{2}$ we first make a change of variable $z \mapsto-1 / z$ in the integrals $\int_{0}^{\infty} z^{j} S_{i}(z) d z$ first. Then the evaluation is done in the same way as above. We find

$$
\int_{0}^{i \infty} z^{j} S_{1}(z) d z=-\frac{2 \pi i N^{\tilde{n}-j}}{\tilde{j}+1}\left(\begin{array}{c}
\tilde{j}+1 \\
n
\end{array}\right) B_{\tilde{j}-n+1} \sum_{\substack{a d=m, a>0 \\
\operatorname{gcd}(a, N)=1}} \frac{1}{a^{\tilde{n}+1} d^{\tilde{j}+1}}
$$

and

$$
\int_{0}^{i \infty} z^{j} S_{2}(z) d z= \begin{cases}0, & \text { if } j<\tilde{n}-1 \\ \frac{2 \pi i}{\tilde{n}} \sum_{\substack{a d=m, a>0 \\ \operatorname{gcd}(a, N)=1}} \frac{1}{a^{\tilde{n}} d^{n+1}}, & \text { if } j=\tilde{n}+1\end{cases}
$$

We then insert the all the estimates above into the definition of $r^{-}\left(R_{\Gamma_{0}(N), w, n}^{m}\right)(X)$. After simplification we arrive at the claimed formula. 


\section{Proof of Theorems 1.4, 1.6, And 1.7}

We first give a proof of Theorems 1.4 .

Proof of Theorems 1.4. Write $d=d_{w}$. To prove that

$$
\left\{R_{\Gamma_{0}(2), w, 2 i} \mid i=1, \ldots, d_{w}\right\}
$$

is a basis, it suffices to prove that the polynomials

$$
r^{-}\left(R_{\Gamma_{0}(2), w, 2 i}\right)(X), \quad i=1,2, \ldots, d
$$

are linearly independent over $\mathbb{C}$.

By Theorem 1.1, for $i=1,2, \ldots, d$, the coefficients of $X^{w-2 j+1}, j=1,2 \ldots, d$, in $r^{-}\left(R_{\Gamma_{0}(2), w, 2 i}\right)(X)$ is

$$
\frac{2^{w-2 i-2 j+1}}{w-2 i+1}\left(\begin{array}{c}
w-2 i+1 \\
2 j-1
\end{array}\right) B_{w-2 i-2 j+2} .
$$

Therefore, the proof of the theorem reduces to that of

$$
\operatorname{det}_{1 \leq i, j \leq d}\left[\frac{2^{w-2 i-2 j+1}}{w-2 i+1}\left(\begin{array}{c}
w-2 i+1 \\
2 j-1
\end{array}\right) B_{w-2 i-2 j+2}\right] \neq 0 .
$$

This would follow immediately from formulas in Theorem A of Appendix A. Here we provide an alternative proof that does not require more advanced tools from the theory of continued fractions.

Using (2.3) we find

$$
\frac{2^{w-2 i-2 j+1}}{w-2 i+1}\left(\begin{array}{c}
w-2 i+1 \\
2 j-1
\end{array}\right) B_{w-2 i-2 j+2}=\frac{(-1)^{w / 2-i-j}}{\pi^{w-2 i-2 j+2}} \frac{(w-2 i) !}{(2 j-1) !} \zeta(w-2 i-2 j+2)
$$

and consequently the determinant in (4.1) is equal to

$$
C \operatorname{det}_{1 \leq i, j \leq d}[\zeta(w-2 i-2 j+2)]
$$

for some non-zero number $C$. Now we have

$$
\begin{aligned}
\operatorname{det}_{1 \leq i, j \leq d}[\zeta(w-2 i-2 j+2)] & =\sum_{m_{1}, \ldots, m_{d}=1}^{\infty} \operatorname{det}_{1 \leq i, j \leq d}\left[m_{i}^{-w+2 i+2 j-2}\right] \\
& =\sum_{m_{1}, \ldots, m_{d}=1}^{\infty} \operatorname{det}_{1 \leq i, j \leq d}\left[m_{i}^{2 j-2}\right] \prod_{i=1}^{d} m_{i}^{-w+2 i} .
\end{aligned}
$$

By the Vandermonde identity, the last expression is equal to

$$
\sum_{m_{1}, \ldots, m_{d}=1}^{\infty} \prod_{1 \leq i<j \leq d}\left(m_{i}^{2}-m_{j}^{2}\right) \prod_{i=1}^{d} m_{i}^{-w+2 i} .
$$

This sum can be alternatively written as

$$
\sum_{1 \leq m_{1}<\ldots<m_{d}} \prod_{1 \leq i<j \leq d}\left(m_{i}^{2}-m_{j}^{2}\right) \sum_{\sigma \in S_{d}} \varepsilon(\sigma) \prod_{i=1}^{d} m_{\sigma(i)}^{-w+2 i},
$$

where the inner sum runs over all elements in the permutation group $S_{d}$, and $\varepsilon(\sigma)$ denotes +1 or -1 according as the permutation $\sigma$ is even or odd. Observe that this inner sum is in fact the expansion of the determinant

$$
\underset{1 \leq i, j \leq d}{\operatorname{det}}\left[m_{j}^{-w+2 i}\right] \text {. }
$$


Using the Vandermonde identity again, we evaluate the above determinant as

$$
\prod_{1 \leq i<j \leq d}\left(m_{i}^{2}-m_{j}^{2}\right) \prod_{i=1}^{d} m_{i}^{2-w}
$$

Therefore,

$$
\operatorname{det}_{1 \leq i, j \leq d}[\zeta(w-2 i-2 j+2)]=\sum_{1 \leq m_{1}<\ldots<m_{d}} \prod_{1 \leq i<j \leq d}\left(m_{i}^{2}-m_{j}^{2}\right)^{2} \prod_{i=1}^{d} m_{i}^{2-w} .
$$

Since the summands above are all strictly positive, this implies that (4.1) holds. This proves that $\left\{R_{\Gamma_{0}(2), w, 2 i} \mid i=1, \ldots, d_{w}\right\}$ is a basis.

The proofs of the other three cases are similar. For the set $\left\{R_{\Gamma_{0}(2), w, w-2 i} \mid i=\right.$ $\left.1, \ldots, d_{w}\right\}$, we find that the coefficients of $X^{2 j-1}$ in $r^{-}\left(R_{\Gamma_{0}(2), w, w-2 i}\right)(X)$ is

$$
-\frac{1}{w-2 i+1}\left(\begin{array}{c}
w-2 i+1 \\
2 j-1
\end{array}\right) B_{w-2 i-2 j+2} \text {. }
$$

By the same argument as before, we see that $R_{\Gamma_{0}(2), w, w-2 i}, i=1, \ldots, d_{w}$ are linearly independent. For the cases $\left\{R_{\Gamma_{0}(2), w, 2 i-1}\right\}$ and $\left\{R_{\Gamma_{0}(2), w, w-2 i+1}\right\}$ we consider the coefficients of $X^{w-2 j}$ and $X^{2 j}, j=1, \ldots, d_{w}$, respectively, in $r^{+}\left(R_{\Gamma_{0}(2), w, 2 i-1}\right)(X)$ and $r^{+}\left(R_{\Gamma_{0}(2), w, w-2 i+1}\right)(X)$. The details are omitted.

Proof of Theorem 1.6. Write $k=w+2$. Let $S_{k}^{\text {old }}\left(\Gamma_{0}(2)\right)$ and $S_{k}^{\text {new }}\left(\Gamma_{0}(2)\right)$ be the spaces of oldforms and newforms of weight $k$ on $\Gamma_{0}(2)$, respectively. Let $d_{k}^{\prime}=\operatorname{dim} S_{k}\left(S L_{2}(\mathbb{Z})\right)$, and $f_{1}, \ldots, f_{d_{k}^{\prime}}$ be the normalized Hecke eigenforms spanning $S_{k}\left(S L_{2}(\mathbb{Z})\right)$. Then the functions

$$
g_{i}=f_{i}+\left.f_{i}\right|_{\omega_{2}}, \quad g_{d_{k}^{\prime}+i}=f_{i}-\left.f_{i}\right|_{\omega_{2}}, \quad i=1, \ldots, d_{k}^{\prime},
$$

form an orthogonal basis for $S_{k}^{\text {old }}\left(\Gamma_{0}(2)\right)$. Let also $g_{2 d_{k}^{\prime}+1}, \ldots, g_{d_{w}}$ be the normalized Hecke eigenforms in $S_{k}^{\text {new }}\left(\Gamma_{0}(2)\right)$. Then the set $\left\{g_{i} \mid i=1, \ldots, d_{w}\right\}$ is an orthogonal basis for $S_{k}\left(\Gamma_{0}(2)\right)$. It follows that

$$
E_{2 j}^{0} E_{k-2 j}^{i \infty}=\sum_{i=1}^{d_{w}} \frac{\left(E_{2 j}^{0} E_{k-2 j}^{i \infty}, g_{i}\right)}{\left(g_{i}, g_{i}\right)} g_{i}
$$

and to show that $\left\{E_{2 j}^{0} E_{k-2 j}^{i \infty}\right\}$ and $\left\{E_{k-2 j}^{0} E_{2 j}^{i \infty}\right\}$ are bases, it suffices to prove that

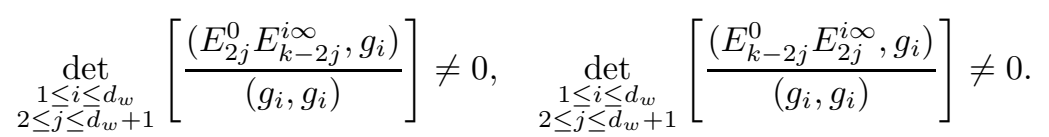

Now according to Proposition 2 of [10], we have, for $i=1, \ldots, 2 d_{k}^{\prime}$,

$$
\begin{aligned}
\left(E_{2 j}^{0} E_{k-2 j}^{i \infty}, g_{i}\right) & =\left(E_{2 j}^{0} E_{k-2 j}^{i \infty}, f_{i} \pm\left. f_{i}\right|_{\omega_{2}}\right) \\
& =c_{j}\left(1+2^{1-k}\left(1-\lambda_{i}\right)\right) L\left(f_{i}, k-1\right) L\left(f_{i} \pm\left. f_{i}\right|_{\omega_{2}}, k-2 j\right) \\
& =c_{j}\left(1+2^{1-k}\left(1-\lambda_{i}\right)\right) L\left(f_{i}, k-1\right) L\left(g_{i}, k-2 j\right),
\end{aligned}
$$

and, for $i=2 d_{k}^{\prime}+1, \ldots, d_{w}$,

$$
\left(E_{2 j}^{0} E_{k-2 j}^{i \infty}, g_{i}\right)=c_{j}\left(\epsilon_{i}+2^{-k / 2}\right) L\left(g_{i}, k-1\right) L\left(g_{i}, k-2 j\right)
$$


where $L(f, s)$ is the Hecke $L$-function associated with an eigenform $f$,

$$
c_{j}=\frac{(k-2) !}{(4 \pi)^{k-1}} \cdot \frac{4 j}{B_{2 j}} \cdot \frac{2^{j}}{1-2^{2 j}} \cdot \frac{1}{\left(1-2^{2 j-k}\right) \zeta(k-2 j)},
$$

$\lambda_{i}=\lambda_{d_{k}^{\prime}+i}$ is the eigenvalue of $f_{i}$ under the Hecke operator $T_{2}$, and $\epsilon_{i}$ is the eigenvalues of $g_{i}$ under the Atkin-Lehner involution $\omega_{2}$. For convenience, we have also set $f_{i}=f_{i-d_{k}^{\prime}}$ for $i=d_{k}^{\prime}+1, \ldots, 2 d_{k}^{\prime}$. It follows that

$$
\operatorname{det}_{\substack{1 \leq i \leq d_{w} \\ 2 \leq j \leq d_{w}+1}}\left[\frac{\left(E_{2 j}^{0} E_{k-2 j}^{i \infty}, g_{i}\right)}{\left(g_{i}, g_{i}\right)}\right]=\operatorname{det}_{\substack{1 \leq i \leq d_{w} \\ 2 \leq j \leq d_{w}+1}}\left[L\left(g_{i}, k-2 j\right)\right] \cdot \prod_{i=1}^{d_{w}} \frac{b_{i}}{\left(g_{i}, g_{i}\right)} \cdot \prod_{j=2}^{d_{w}+1} c_{j}
$$

and

$$
\operatorname{det}_{\substack{1 \leq i \leq d_{w} \\ 2 \leq j \leq d_{w}+1}}\left[\frac{\left(E_{k-2 j}^{0} E_{2 j}^{i \infty}, g_{i}\right)}{\left(g_{i}, g_{i}\right)}\right]=\operatorname{det}_{\substack{1 \leq i \leq d_{w} \\ 2 \leq j \leq d_{w}+1}}\left[L\left(g_{i}, 2 j\right)\right] \cdot \prod_{i=1}^{d_{w}} \frac{b_{i}}{\left(g_{i}, g_{i}\right)} \cdot \prod_{j=2}^{d_{w}+1} c_{k / 2-j}
$$

where

$$
b_{i}= \begin{cases}\left(1+2^{1-k}\left(1-\lambda_{i}\right)\right) L\left(f_{i}, k-1\right), & \text { for } i=1, \ldots, 2 d_{k}^{\prime}, \\ \left(\epsilon_{i}+2^{-k / 2}\right) L\left(g_{i}, k-1\right), & \text { for } i=2 d_{k}^{\prime}+1, \ldots, d_{w} .\end{cases}
$$

Clearly, the numbers $c_{j}$ are never zero. Also, using the upper bound $\left|a_{p}\right| \leq 2 p^{(k-1) / 2}$ for the $p$-th Fourier coefficients of a normalized Hecke eigenform $\sum a_{n} q^{n}$ on $S L_{2}(\mathbb{Z})$, we know that $L\left(f_{i}, k-1\right), L\left(g_{i}, k-1\right)$ and $\left(1+2^{1-k}\left(1-\lambda_{i}\right)\right)$ do not vanish. Then the proof of the theorem reduces to that of

$$
\operatorname{det}_{\substack{1 \leq i \leq d_{w} \\ 2 \leq j \leq d_{w}+1}}\left[L\left(g_{i}, k-2 j\right)\right] \neq 0, \quad \operatorname{det}_{\substack{1 \leq i \leq d_{w} \\ 2 \leq j \leq d_{w}+1}}\left[L\left(g_{i}, 2 j\right)\right] \neq 0 .
$$

On the other hand, consider the sets

$$
\left\{R_{\Gamma_{0}(2), w, 2 j+1} \mid j=1, \ldots, d_{w}\right\}, \quad\left\{R_{\Gamma_{0}(2), w, w-2 j-1} \mid j=1, \ldots, d_{w}\right\} .
$$

Again, we have

$$
R_{\Gamma_{0}(2), w, n}=\sum_{i=1}^{d_{w}} \frac{\left(R_{\Gamma_{0}(2), w, n}, g_{i}\right)}{\left(g_{i}, g_{i}\right)} g_{i}
$$

By Definition 1.1.

$$
2^{-1}(2 i)^{w+1}\left(g_{i}, R_{\Gamma_{0}(2), w, n}\right)=\int_{0}^{i \infty} g_{i}(z) z^{n} d z=\frac{n !}{(-2 \pi i)^{n+1}} L\left(g_{i}, n+1\right) .
$$

It follows that (4.2) holds if and only if the two sets in (4.3) are both bases for $S_{w+2}\left(\Gamma_{0}(2)\right)$.

To show that

$$
\left\{R_{\Gamma_{0}(2), w, 2 j+1} \mid j=1, \ldots, d_{w}\right\}
$$

is a basis, we consider the coefficients of $X^{w-2 i}$ in $r^{+}\left(R_{\Gamma_{0}(2), w, 2 j+1}\right)$. When $w$ is a multiple of 4 , the coefficients are

$$
\frac{2^{w-2 i-2 j-1}}{w-2 j}\left(\begin{array}{c}
w-2 j \\
2 i
\end{array}\right) B_{w-2 i-2 j},
$$


and the proof proceeds as in Theorem 1.4, and the details are skipped. When $w=4 d+2$ with $d_{w}=d$ for some positive integer $d$, the coefficients are

$$
a_{i j}=\frac{2^{w-2 i-2 j-1}}{w-2 j}\left(\begin{array}{c}
w-2 j \\
2 i
\end{array}\right) B_{w-2 i-2 j}-\delta_{d, i} \delta_{d, j} \frac{B_{0}}{2 d+2} .
$$

Then we have

$$
\begin{aligned}
\operatorname{det}_{1 \leq i, j \leq d}\left[a_{i j}\right]=\operatorname{det}_{1 \leq i, j \leq d} & {\left[\frac{2^{w-2 i-2 j-1}}{w-2 j}\left(\begin{array}{c}
w-2 j \\
2 i
\end{array}\right) B_{w-2 i-2 j}\right] } \\
& -\frac{1}{2 d+2} \operatorname{det}_{1 \leq i, j \leq d-1}\left[\frac{2^{w-2 i-2 j-1}}{w-2 j}\left(\begin{array}{c}
w-2 j \\
2 i
\end{array}\right) B_{w-2 i-2 j}\right] .
\end{aligned}
$$

By the first and the third identities of Theorem A in Appendix A, the first term is equal to

$$
\begin{aligned}
2^{d(w-1)} & \left(\prod_{i=1}^{d} 2^{-2 i}\right)^{2} \prod_{j=1}^{d}(w-2 j-1) ! \prod_{i=1}^{d} \frac{1}{(2 i) !} \operatorname{det}_{1 \leq i, j \leq d}\left[\frac{B_{w-2 i-2 j}}{(w-2 i-2 j) !}\right] \\
= & 2^{-d} \prod_{i=1}^{d} \frac{(w-2 i-1) !}{(2 i) !} \prod_{i=1}^{2 d-1}(2 i+1)^{-(2 d-i)},
\end{aligned}
$$

while the second term is

$$
\begin{gathered}
\frac{2^{(d-1)(w-1)}}{d+2}\left(\prod_{i=1}^{d-1} 2^{-2 i}\right)^{2} \prod_{j=1}^{d-1}(w-2 j-1) ! \prod_{i=1}^{d-1} \frac{1}{(2 i) !} \operatorname{det}_{1 \leq i, j \leq d-1}\left[\frac{B_{w-2 i-2 j}}{(w-2 i-2 j) !}\right] \\
=\frac{2^{1-d}}{2 d+2} \prod_{i=1}^{d-1} \frac{(w-2 i-1) !}{(2 i) !} d(2 d+1) \prod_{i=1}^{2 d-1}(2 i+1)^{-(2 d-i)} .
\end{gathered}
$$

The ratio of these two terms is

$$
\frac{d+1}{d(2 d+1)} \frac{(w-2 d-1) !}{(2 d) !}=\frac{d+1}{d}
$$

which is never equal to 1 . From this we conclude that $\operatorname{det}_{1 \leq i, j \leq d}\left[a_{i j}\right] \neq 0$ and thus that $\left\{E_{2 j+2}^{0} E_{w-2 j}^{i \infty} \mid j=1, \ldots, d_{w}\right\}$ is a basis. The proof of the assertion about $\left\{E_{2 j+2}^{i \infty} E_{w-2 j}^{0} \mid j=1, \ldots, d_{w}\right\}$ is similar, and is omitted here.

Proof of Theorem 1.7. The proof uses a straightforward argument in elementary linear algebra, and is skipped here. For more details, see Theorem 2.9 of $[8]$.

\section{Acknowledgment}

The authors would like to thank Professor C. Krattenthaler for his permission to include his proof of formulas for Hankel determinants of Bernoulli numbers in the paper. The authors would also like to thank Professors H. H. Chan and N. Yui for reading earlier versions of the paper and giving valuable comments. Finally, the authors would like to express their gratitude to the anonymous referee for thorough reading of the manuscript.

Yifan Yang was supported by Grant 95-2115-M-009-005 of the National Science Council (NSC) of the Republic of China (Taiwan). Part of this work was done while he was visiting Tsuda College, Japan. He would like to thank Tsuda College for the enormous hospitality. 


\section{Appendix A: Evaluation of Hankel determinants of Bernoulli numbers} by Christian Krattenthaler

In this appendix we will derive exact formulas for Hankel determinants formed by Bernoulli numbers. We utilize the following theorem.

Theorem CF. Let $\left(\mu_{k}\right)_{k \geq 0}$ be a sequence with generating function $\sum_{k=0}^{\infty} \mu_{k} x^{k}$ written in the form

Then we have

$$
\sum_{k=0}^{\infty} \mu_{k} x^{k}=\frac{\mu_{0}}{1+\frac{a_{1} x}{1+\frac{a_{2} x}{1+\ddots}}}
$$

$$
\begin{aligned}
& \operatorname{det}_{0 \leq i, j \leq n-1}\left[\mu_{i+j}\right]=\mu_{0}^{n}\left(a_{1} a_{2}\right)^{n-1}\left(a_{3} a_{4}\right)^{n-2} \ldots\left(a_{2 n-5} a_{2 n-4}\right)^{2}\left(a_{2 n-3} a_{2 n-2}\right), \\
& \operatorname{det}_{0 \leq i, j \leq n-1}\left[\mu_{i+j+1}\right]=(-1)^{n} \mu_{0}^{n} a_{1}^{n}\left(a_{2} a_{3}\right)^{n-1}\left(a_{4} a_{5}\right)^{n-2} \ldots\left(a_{2 n-4} a_{2 n-3}\right)^{2}\left(a_{2 n-2} a_{2 n-1}\right), \\
& \text { and } \operatorname{det}_{0 \leq i, j \leq n-1}\left[\mu_{i+j+2}\right]=\mu_{0}^{n} a_{1}^{n}\left(a_{2} a_{3}\right)^{n-1}\left(a_{4} a_{5}\right)^{n-2} \ldots\left(a_{2 n-4} a_{2 n-3}\right)^{2}\left(a_{2 n-2} a_{2 n-1}\right) \\
& \times \sum_{0 \leq i_{1}-1<i_{2}-2<\ldots<i_{n}-n \leq n} a_{i_{1}} a_{i_{2}} \ldots a_{i_{n}} .
\end{aligned}
$$

Here the first two formulas are from Theorem 7.2 of [11], and the third is from Theorem 30 of [13].

Theorem A. Let $B_{n}$ denote the $n$-th Bernoulli number. Then we have

$$
\begin{aligned}
& \underset{0 \leq i, j \leq n-1}{\operatorname{det}}\left[\frac{B_{2 i+2 j+2}}{(2 i+2 j+2) !}\right]=4^{-n^{2}} \prod_{i=1}^{2 n-1}(2 i+1)^{-(2 n-i)}, \\
& \underset{0 \leq i, j \leq n-1}{\operatorname{det}}\left[\frac{B_{2 i+2 j+4}}{(2 i+2 j+4) !}\right]=(-1)^{n} 4^{-n^{2}-n} 9^{-n} \prod_{i=1}^{2 n-1}(2 i+3)^{-(2 n-i)}, \\
& \operatorname{det}_{0 \leq i, j \leq n-1}\left[\frac{B_{2 i+2 j+6}}{(2 i+2 j+6) !}\right]=4^{-n^{2}-2 n}(n+1)(2 n+3) \prod_{i=1}^{2 n+1}(2 i+1)^{-(2 n+2-i)} .
\end{aligned}
$$

Proof. In view of Theorem CF, we should determine the continued fraction expansion for the generating function for the numbers $B_{2 n} /(2 n) !, n \geq 1$. Writing $t=x / 2$, we have

$$
\begin{aligned}
\sum_{k \geq 0} \frac{B_{k+2}}{(k+2) !} x^{k} & =\frac{1}{x^{2}}\left(\frac{x}{e^{x}-1}-1+\frac{x}{2}\right)=\frac{1}{4 t^{2}} \frac{t e^{t}+t e^{-t}-e^{t}+e^{-t}}{e^{t}-e^{-t}} \\
& =\frac{1}{4 t^{2}} \frac{\sum_{n \geq 1}(2 n) \frac{t^{2 n+1}}{(2 n+1) !}}{\sum_{n \geq 0} \frac{t^{2 n+1}}{(2 n+1) !}}=\frac{1}{12} \frac{{ }_{0} F_{1}\left(-; \frac{5}{2} ; \frac{t^{2}}{4}\right)}{{ }_{0} F_{1}\left(-; \frac{3}{2} ; \frac{t^{2}}{4}\right)}
\end{aligned}
$$


Equivalently, by using the fact that $B_{n}=0$ for odd $n \geq 3$,

$$
\sum_{k \geq 0} \frac{B_{2 k+2}}{(2 k+2) !} x^{k}=\frac{1}{12} \frac{{ }_{0} F_{1}\left(-; \frac{5}{2} ; \frac{x}{16}\right)}{{ }_{0} F_{1}\left(-; \frac{3}{2} ; \frac{x}{16}\right)} .
$$

Now, Gauss proved the following continued fraction expansion

$$
\frac{{ }_{2} F_{1}(a, b+1 ; c+1 ; z)}{{ }_{2} F_{1}(a, b ; c ; z)}=\frac{1}{1+\frac{a_{1} z}{1+\frac{a_{2} z}{1+\ddots}}},
$$

where

$$
a_{2 n-1}=-\frac{(a+n-1)(c-b+n-1)}{(c+2 n-2)(c+2 n-1)}, \quad a_{2 n}=-\frac{(b+n)(c-a+n)}{(c+2 n-1)(c+2 n)} .
$$

(See [11, Theorem 6.1].) Replacing $a$ by $b, c$ by $3 / 2$, and $z$ by $x^{2} /\left(16 b^{2}\right)$ and subsequently letting $b$ tend to infinity, we obtain

$$
\frac{{ }_{0} F_{1}\left(-; \frac{5}{2} ; \frac{x}{16}\right)}{{ }_{0} F_{1}\left(-; \frac{3}{2} ; \frac{x}{16}\right)}=\frac{1}{1+\frac{a_{1} x}{1+\frac{a_{2} x}{1+\ddots}}}
$$

where

$$
a_{2 n-1}=\frac{1}{4(4 n-1)(4 n+1)}, \quad a_{2 n}=\frac{1}{4(4 n+1)(4 n+3)},
$$

that is,

$$
a_{n}=\frac{1}{4(2 n+1)(2 n+3)} .
$$

We now apply Theorem CF with $\mu_{k}=B_{2 k+2} /(2 k+2)$ !. The first identity of the theorem yields

$$
\begin{aligned}
\operatorname{det}_{0 \leq i, j \leq n-1}\left[\frac{B_{2 i+2 j+2}}{(2 i+2 j+2) !}\right] & =12^{-n} \prod_{k=1}^{n-1}\left(16(4 k-1)(4 k+1)^{2}(4 k+3)\right)^{-(n-k)} \\
& =4^{-n^{2}} 3^{-n}\left(3^{-(n-1)} 5^{-(2 n-2)} \ldots(4 n-3)^{2}(4 n-1)\right) \\
& =4^{-n^{2}} \prod_{i=1}^{2 n-1}(2 i+1)^{-(2 n-i)},
\end{aligned}
$$

and the second identity gives

$$
\begin{aligned}
\operatorname{det}_{0 \leq i, j \leq n-1}\left[\frac{B_{2 i+2 j+4}}{(2 i+2 j+4) !}\right] & =(-720)^{-n} \prod_{k=1}^{n-1}\left(16(4 k+1)(4 k+3)^{2}(4 k+5)\right)^{-(n-k)} \\
& =(-1)^{n} 4^{-n^{2}-n} 9^{-n} \prod_{i=1}^{2 n-1}(2 i+3)^{-(2 n-i)}
\end{aligned}
$$


For the third case, we have

$$
\begin{aligned}
\underset{0 \leq i, j \leq n-1}{\operatorname{det}}\left[\frac{B_{2 i+2 j+6}}{(2 i+2 j+6) !}\right]=(-1)^{n} & \operatorname{det}_{0 \leq i, j \leq n-1}\left[\frac{B_{2 i+2 j+4}}{(2 i+2 j+4) !}\right] \\
& \times \sum_{0 \leq i_{1}-1<i_{2}-2<\ldots<i_{n}-n \leq n} a_{i_{1}} \ldots a_{i_{n}} .
\end{aligned}
$$

Now

$$
\begin{aligned}
& \sum_{0 \leq i_{1}-1<i_{2}-2<\ldots<i_{n}-n \leq n} a_{i_{1}} \ldots a_{i_{n}} \\
= & \sum_{0 \leq i_{1}-1<\ldots<i_{n}-n \leq n} \frac{1}{\left(2 i_{1}+1\right)\left(2 i_{1}+3\right)\left(2 i_{2}+1\right)\left(2 i_{2}+3\right) \ldots\left(2 i_{n}+1\right)\left(2 i_{n}+3\right)} .
\end{aligned}
$$

The denominator of each summand is a product of $2 n$ distinct odd integers among the $2 n+1$ odd integers ranging from 3 to $4 n+3$. The only missing odd integer must be of the form $4 k+3$, corresponding to the choice $\left(i_{1}, \ldots, i_{n}\right)=(1,3, \ldots, 2 k-$ $1,2 k+2, \ldots, 2 n-2,2 n)$. Thus, we see that

$$
\sum_{0 \leq i_{1}-1<\ldots<i_{n}-n \leq n} a_{i_{1}} \ldots a_{i_{n}}=\frac{1}{4^{n}(4 n+3) ! !} \sum_{k=0}^{n}(4 k+3)=\frac{(2 n+3)(n+1)}{4^{n}(4 n+3) ! !},
$$

where $(4 n+3)$ !! denotes the product of all odd integers less than or equal to $4 n+3$. After simplification, we arrive at the third identity. This completes the proof.

\section{Appendix B: Computing matrices representing the Hecke operators on} $S_{w+2}\left(\Gamma_{0}(2)\right)$ and their characteristic polynomials

We will demonstrate a Mathematica 11 program which, for given $w$ and $m$, yields a matrix representing the Hecke operator $T_{m}$ on $S_{w+2}\left(\Gamma_{0}(2)\right)$, and its characteristic polynomial. The program is a straightforward application of Theorem 1.7.

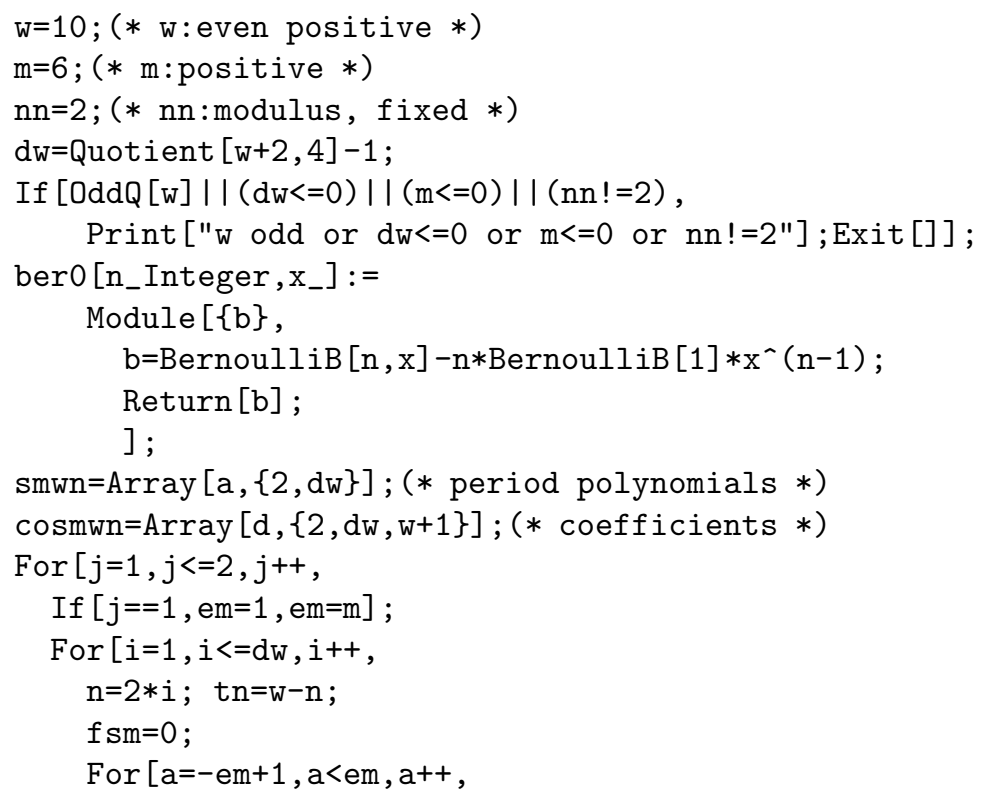

\footnotetext{
${ }^{1}$ Mathematica is a trademark of Wolfram Research, Inc.
} 


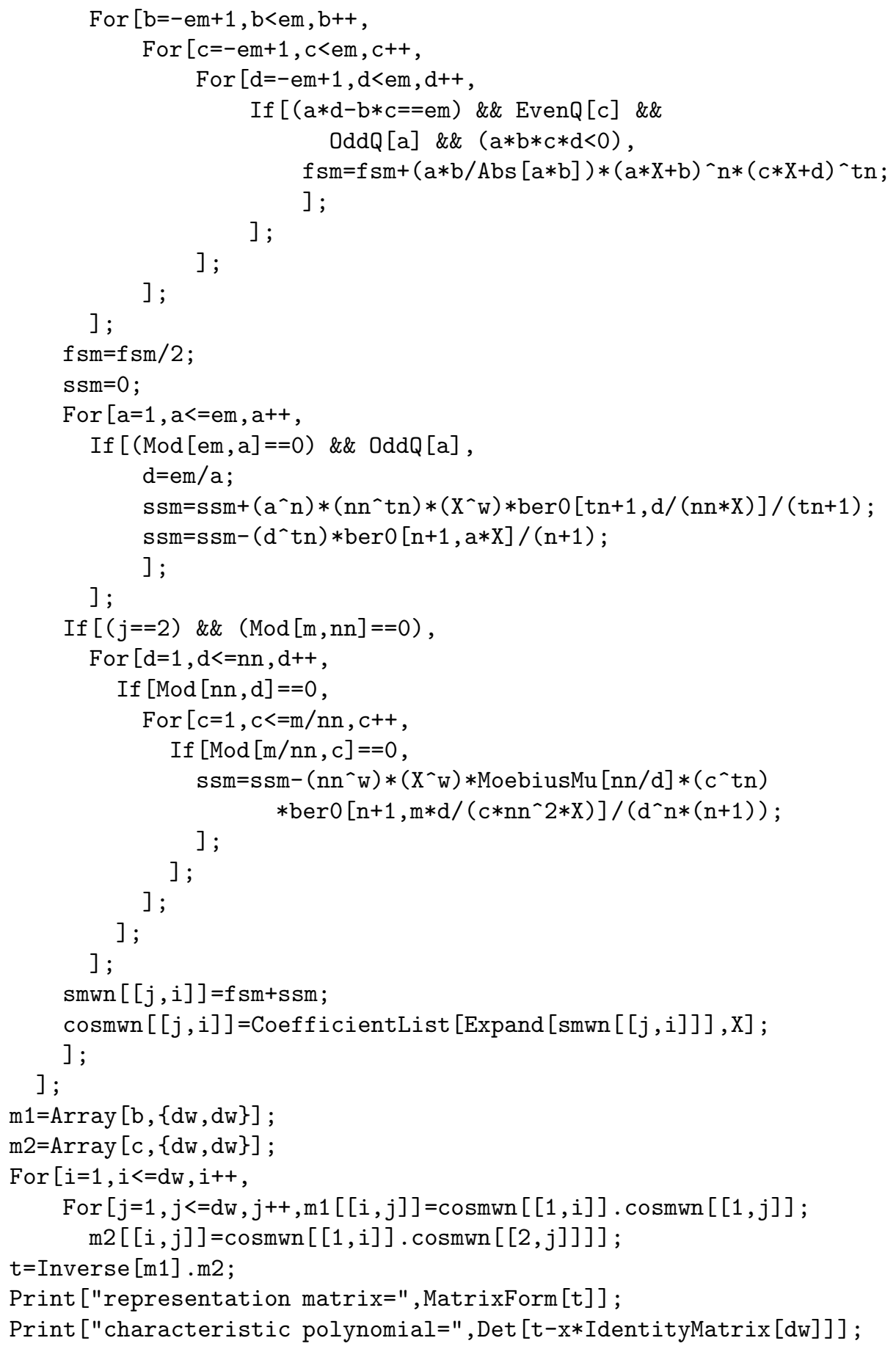

The reader might be interested in comparing these results with those given at "The Modular Form Database" (http://modular.fas.harvard.edu/Tables/tables.html) by William Stein. 


\section{REFERENCES}

[1] Antoniadis, J.A.: Modulformen auf $\Gamma_{0}(N)$ mit rationalen Perioden. Manuscripta Math. 74(4), 359-384 (1992)

[2] Chan, H.H., Chua, K.S.: Representations of integers as sums of 32 squares. Ramanujan J. 7(1-3), 79-89 (2003). Rankin memorial issues

[3] Cohen, H.: Sums involving the values at negative integers of $L$-functions of quadratic characters. Math. Ann. 217(3), 271-285 (1975)

[4] Cohen, H.: Sur certaines sommes de séries liées aux périodes de formed modulaires (1981)

[5] Diamantis, N.: Hecke operators and derivatives of $L$-functions. Compositio Math. 125(1), 39-54 (2001)

[6] Diamond, F., Shurman, J.: A first course in modular forms, Graduate Texts in Mathematics, vol. 228. Springer-Verlag, New York (2005)

[7] Eichler, M.: Eine Verallgemeinerung der Abelschen Integrale. Math. Z. 67, 267-298 (1957)

[8] Fukuhara, S.: Explicit formulas for Hecke operators on cusp forms, Dedekind symbols and period polynomials. J. Reine Angew. Math. (to appear), arXiv:math.NT/0506373

[9] Fukuhara, S.: Hecke operators on weighted Dedekind symbols. J. Reine Angew. Math. 593, $1-29(2006)$

[10] Imamoḡlu, Ö., Kohnen, W.: Representations of integers as sums of an even number of squares. Math. Ann. 333(4), 815-829 (2005)

[11] Jones, W.B., Thron, W.J.: Continued fractions, Encyclopedia of Mathematics and its Applications, vol. 11. Addison-Wesley Publishing Co., Reading, Mass. (1980). Analytic theory and applications, With a foreword by Felix E. Browder, With an introduction by Peter Henrici

[12] Kohnen, W., Zagier, D.: Modular forms with rational periods. In: Modular forms (Durham, 1983), Ellis Horwood Ser. Math. Appl.: Statist. Oper. Res., pp. 197-249. Horwood, Chichester (1984)

[13] Krattenthaler, C.: Advanced determinant calculus: a complement. Linear Algebra Appl. 411, 68-166 (2005)

[14] Manin, Y.I.: Explicit formulas for the eigenvalues of Hecke operators. Acta Arith. 24, 239249 (1973). Collection of articles dedicated to Carl Ludwig Siegel on the occasion of his seventy-fifth birthday. III

[15] Manin, Y.I.: Periods of cusp forms, and p-adic Hecke series. Mat. Sb. (N.S.) 92(134), 378401, 503 (1973)

[16] Rankin, R.A.: The construction of automorphic forms from the derivatives of a given form. J. Indian Math. Soc. (N.S.) 20, 103-116 (1956)

[17] Shimura, G.: Sur les intégrales attachées aux formes automorphes. J. Math. Soc. Japan 11, 291-311 (1959)

[18] Skoruppa, N.P.: Binary quadratic forms and the Fourier coefficients of elliptic and Jacobi modular forms. J. Reine Angew. Math. 411, 66-95 (1990)

[19] Zagier, D.: Hecke operators and periods of modular forms. In: Festschrift in honor of I. I. Piatetski-Shapiro on the occasion of his sixtieth birthday, Part II (Ramat Aviv, 1989), Israel Math. Conf. Proc., vol. 3, pp. 321-336. Weizmann, Jerusalem (1990)

[20] Zagier, D.: Periods of modular forms and Jacobi theta functions. Invent. Math. 104(3), 449-465 (1991)

Department of Mathematics, Tsuda College, Tsuda-machi 2-1-1, Kodaira-Shi, Tokyo 187-8577, JAPAN

E-mail address: fukuhara@tsuda.ac.jp

Department of Applied Mathematics, National Chiao Tung University, 1001 Ta Hsueh

Road, HSinchu, TAIWAN 300

E-mail address: yfyang@math.nctu.edu.tw 